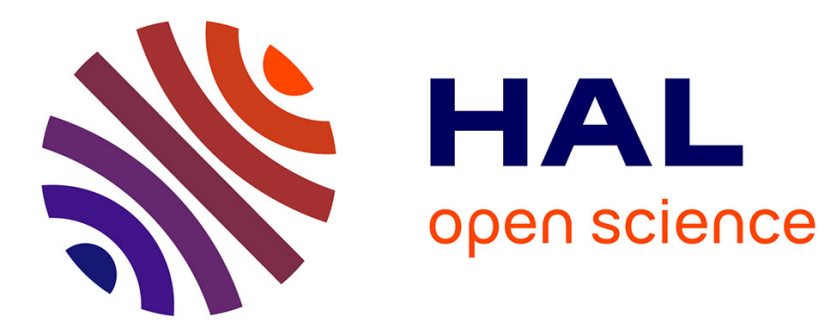

\title{
Quelle incidence des médicaments sur les accidents de la route et sur les délits routiers? Analyse d'une série de cas au CHU de Rouen
}

\author{
Maxime Thiollent
}

\section{- To cite this version:}

Maxime Thiollent. Quelle incidence des médicaments sur les accidents de la route et sur les délits routiers? Analyse d'une série de cas au CHU de Rouen. Sciences pharmaceutiques. 2018. dumas02086672

\section{HAL Id: dumas-02086672 https://dumas.ccsd.cnrs.fr/dumas-02086672}

Submitted on 1 Apr 2019

HAL is a multi-disciplinary open access archive for the deposit and dissemination of scientific research documents, whether they are published or not. The documents may come from teaching and research institutions in France or abroad, or from public or private research centers.
L'archive ouverte pluridisciplinaire HAL, est destinée au dépôt et à la diffusion de documents scientifiques de niveau recherche, publiés ou non, émanant des établissements d'enseignement et de recherche français ou étrangers, des laboratoires publics ou privés. 


\section{UNIVERSITE DE ROUEN}

\section{UFR DE MEDECINE ET DE PHARMACIE}

2018

$\mathrm{N}^{\circ}$

\section{THESE}

pour le DIPLOME D'ETAT DE DOCTEUR EN

PHARMACIE

Présentée et soutenue publiquement le 19 décembre 2018

par

Maxime THIOLLENT

Né le 21 février 1992 à Le Havre

Quelle incidence des médicaments sur les accidents de la route et sur les délits routiers?

Analyse d'une série de cas au CHU de Rouen.

Directeur de thèse : $\quad$ Docteur Fabien LAMOUREUX, PH

Co-directeur de thèse : Professeur Michel GUERBET, PU

Président du jury : $\quad$ Professeur Michel GUERBET, PU

Membres du jury : $\quad$ Docteur Fabien LAMOUREUX, PH

Docteur Marc PONTHIEUX, Pharmacien d'officine

Docteur Laurent IMBERT, PH 



\section{Remerciements}

Je tiens à remercier dans un premier temps, très affectueusement le directeur de cette thèse, le Dr Fabien LAMOUREUX, pour sa gentillesse, sa disponibilité malgré mes nombreuses visites « surprises », son professionnalisme. Mais aussi pour son écoute, son implication dans ce travail, ses très nombreux conseils, sa pédagogie, et enfin son accueil depuis le début lors de mon stage de $5^{\text {ème }}$ année.

Je remercie le Professeur Michel GUERBET pour avoir accepté d'être président du jury mais aussi co-directeur de cette thèse. Je remercie également les autres membres du jury d'avoir accepté d'assister à la présentation de ce travail Marc PONTHIEUX et Laurent IMBERT (ainsi que pour ses nombreux conseils).

A toute l'équipe du laboratoire de Pharmacologie -Toxicologie du CHU de Rouen, Caro, Marina, Sophie, Patricia, Nancy, Tony (le mannequin), David (mon lapin), merci. Merci de votre gentillesse, de votre accueil, de vos sourires, de votre bonne humeur, de vos conseils, de votre aide permanente (surtout pour les analyses) sans vous, ce travail aurait été beaucoup plus laborieux.

Enfin,

A ma famille, ma motivation, mon soutien. Merci à toi marraine, à toi grand-mère pour vos relectures assidues. Merci à vous papa et maman d'être là au quotidien pour moi, de m'avoir permis de faire les études dont je rêvais. Je vous aime.

A vous les amis de longues dates, Mélissandre pour m'avoir maintenu éveillé lors de ces longues soirées, pleins de bonheur avec Victor et le bébé, Thibault, Romain, Etienne pour me permettre de changer d'air lors de nos soirées. 
A Amanda, d'être arrivée dans ma vie au cours de ces études, de m'avoir boosté à travailler ma thèse, formé pour la bibliographie, d'égayer mes journées.

A tous les copains de Pharma-si-si-la-famille, merci pour tous ces incroyables moments passés ensemble, je n'oublierai jamais. Mention spéciale pour les bro': Lolo, Yoyo (mon petit caillot) et Doudou (alias Dieu 73) cœur sur vous.

Pour vous Pépé, Mémé et Mamie. 

« L'Université de Rouen et l'UFR de Médecine et de Pharmacie de Rouen n'entendent donner aucune approbation ni improbation aux opinions émises dans cette thèse. Ces opinions sont propres à leurs auteurs. » 

ANNEE UNIVERSITAIRE 2016 - 2017

U.F.R. DE MEDECINE ET DE-PHARMACIE DE ROUEN

DOYEN :

Professeur Pierre FREGER

ASSESSEURS :

Professeur Michel GUERBET

Professeur Benoit VEBER

Professeur Guillaume SAVOYE

\section{I - MEDECINE}

\section{PROFESSEURS DES UNIVERSITES - PRATICIENS HOSPITALIERS}

Mr Frédéric ANSELME

$\mathrm{HCN}$ Cardiologie

Mme Gisèle APTER

Havre Pédopsychiatrie

Mme Isabelle AUQUIT AUCKBUR

HCN Chirurgie plastique

Mr Fabrice BAUER

HCN Cardiologie

Mme Soumeya BEKRI

$\mathrm{HCN}$ Biochimie et biologie moléculaire

Mr Ygal BENHAMOU

HCN Médecine interne

Mr Jacques BENICHOU

$\mathrm{HCN}$ Bio statistiques et informatique médicale

Mr Olivier BOYER

UFR Immunologie

Mme Sophie CANDON

$\mathrm{HCN}$ Immunologie

Mr François CARON

$\mathrm{HCN}$ Maladies infectieuses et tropicales

Mr Philippe CHASSAGNE(détachement)HCN Médecine interne (gériatrie) - Détachement

Mr Vincent COMPERE

HCN Anesthésiologie et réanimation chirurgicale

Mr Jean-Nicolas CORNU

HCN Urologie

Mr Antoine CUVELIER

HB Pneumologie 
Mr Pierre CZERNICHOW(surnombre) HCH Epidémiologie, économie de la santé

Mr Jean-Nicolas DACHER

$\mathrm{HCN}$ Radiologie et imagerie médicale

Mr Stéfan DARMONI

HCN Informatique médicale et techniques de communication

Mr Pierre DECHELOTTE

HCN Nutrition

Mr Stéphane DERREY

HCN Neurochirurgie

\begin{tabular}{|c|c|c|}
\hline Mr Frédéric DI FIORE & $C B$ & Cancérologie \\
\hline Mr Fabien DOGUET & $\mathrm{HCN}$ & Chirurgie Cardio Vasculaire \\
\hline Mr Jean DOUCET & SJ & Thérapeutique - Médecine interne et gériatrie \\
\hline Mr Bernard DUBRAY & $\mathrm{CB}$ & Radiothérapie \\
\hline Mr Philippe DUCROTTE & $\mathrm{HCN}$ & Hépato-gastro-entérologie \\
\hline Mr Frank DUJARDIN & $\mathrm{HCN}$ & Chirurgie orthopédique - Traumatologique \\
\hline Mr Fabrice DUPARC & $\mathrm{HCN}$ & Anatomie - Chirurgie orthopédique et traumatologique \\
\hline Mr Eric DURAND & $\mathrm{HCN}$ & Cardiologie \\
\hline Mr Bertrand DUREUIL & $\mathrm{HCN}$ & Anesthésiologie et réanimation chirurgicale \\
\hline Mme Hélène ELTCHANINOFF & $\mathrm{HCN}$ & Cardiologie \\
\hline Mr Manuel ETIENNE & \multicolumn{2}{|c|}{ HCNMaladies infectieuses et tropicales } \\
\hline Mr Thierry FREBOURG & UFR & Génétique \\
\hline Mr Pierre FREGER & $\mathrm{HCN}$ & Anatomie - Neurochirurgie \\
\hline Mr Jean François GEHANNO & $\mathrm{HCN}$ & Médecine et santé au travail \\
\hline Mr Emmanuel GERARDIN & $\mathrm{HCN}$ & Imagerie médicale \\
\hline Mme Priscille GERARDIN & $\mathrm{HCN}$ & Pédopsychiatrie \\
\hline M. Guillaume GOURCEROL & $\mathrm{HCN}$ & Physiologie \\
\hline Mr Dominique GUERROT & $\mathrm{HCN}$ & Néphrologie \\
\hline Mr Olivier GUILLIN & $\mathrm{HCN}$ & Psychiatrie Adultes \\
\hline Mr Didier HANNEQUIN & $\mathrm{HCN}$ & Neurologie \\
\hline MrFabriceJARDIN & $C B$ & Hématologie \\
\hline Mr Luc-Marie JOLY & $\mathrm{HCN}$ & Médecine d'urgence \\
\hline Mr Pascal JOLY & $\mathrm{HCN}$ & Dermato-Vénéréologie \\
\hline Mme Bouchra LAMIA & Havre & Pneumologie \\
\hline Mme Annie LAQUERRIERE & $\mathrm{HCN}$ & Anatomie et cytologie pathologiques \\
\hline Mr Vincent LAUDENBACH & $\mathrm{HCN}$ & Anesthésie et réanimation chirurgicale \\
\hline
\end{tabular}




\begin{tabular}{|c|c|c|}
\hline Mr Joël LECHEVALLIER & $\mathrm{HCN}$ & Chirurgie infantile \\
\hline Mr Hervé LEFEBVRE & $\mathrm{HB}$ & Endocrinologie et maladies métaboliques \\
\hline Mr Thierry LEQUERRE & $\mathrm{HB}$ & Rhumatologie \\
\hline Mme Anne-Marie LEROI & $\mathrm{HCN}$ & Physiologie \\
\hline Mr Hervé LEVESQUE & $H B$ & Médecine interne \\
\hline Mme Agnès LIARD-ZMUDA & $\mathrm{HCN}$ & Chirurgie Infantile \\
\hline Mr Pierre Yves LITZLER & $\mathrm{HCN}$ & Chirurgie cardiaque \\
\hline Mr Bertrand MACE & $\mathrm{HCN}$ & Histologie, embryologie, cytogénétique \\
\hline M. David MALTETE & $\mathrm{HCN}$ & Neurologie \\
\hline Mr Christophe MARGUET & $\mathrm{HCN}$ & Pédiatrie \\
\hline Mme Isabelle MARIE & $\mathrm{HB}$ & Médecine interne \\
\hline Mr Jean-Paul MARIE & $\mathrm{HCN}$ & Oto-rhino-laryngologie \\
\hline Mr Loïc MARPEAU & $\mathrm{HCN}$ & Gynécologie - Obstétrique \\
\hline Mr Stéphane MARRET & $\mathrm{HCN}$ & Pédiatrie \\
\hline Mme Véronique MERLE & $\mathrm{HCN}$ & Epidémiologie \\
\hline Mr Pierre MICHEL & $\mathrm{HCN}$ & Hépato-gastro-entérologie \\
\hline M. Benoit MISSET & $\mathrm{HCN}$ & Réanimation Médicale \\
\hline Mr Jean-François MUIR(surnombre) & $\mathrm{HB}$ & Pneumologie \\
\hline Mr Marc MURAINE & $\mathrm{HCN}$ & Ophtalmologie \\
\hline Mr Philippe MUSETTE & $\mathrm{HCN}$ & Dermatologie - Vénéréologie \\
\hline Mr Christophe PEILLON & $\mathrm{HCN}$ & Chirurgie générale \\
\hline Mr Christian PFISTER & $\mathrm{HCN}$ & Urologie \\
\hline Mr Jean-Christophe PLANTIER & $\mathrm{HCN}$ & Bactériologie - Virologie \\
\hline Mr Didier PLISSONNIER & $\mathrm{HCN}$ & Chirurgie vasculaire \\
\hline Mr Gaëtan PREVOST & $\mathrm{HCN}$ & Endocrinologie \\
\hline \multicolumn{2}{|c|}{ Mr Jean-Christophe RICHARD (détachement) $\mathrm{HCN}$} & Réanimation médicale - Médecine d'urgence \\
\hline Mr Vincent RICHARD & UFR & Pharmacologie \\
\hline Mme Nathalie RIVES & $\mathrm{HCN}$ & Biologie du développement et de la reproduction \\
\hline Mr Horace ROMAN & $\mathrm{HCN}$ & Gynécologie - Obstétrique \\
\hline Mr Jean-Christophe SABOURIN & $\mathrm{HCN}$ & Anatomie - Pathologie \\
\hline Mr Guillaume SAVOYE & $\mathrm{HCN}$ & Hépato-gastrologie \\
\hline Mme Céline SAVOYE-COLLET & $\mathrm{HCN}$ & Imagerie médicale \\
\hline
\end{tabular}


Mme Pascale SCHNEIDER

Mr Michel SCOTTE

Mme Fabienne TAMION

Mr LUC THIBERVILLE

Mr Christian THUILLEZ(surnombre)

Mr Hervé TILLY

M. Gilles TOURNEL

Mr Olivier TROST

Mr Jean-Jacques TUECH

Mr Jean-Pierre VANNIER(surnombre)

Mr Benoît VEBER

Mr Pierre VERA

Mr Eric VERIN

Mr Eric VERSPYCK

Mr Olivier VITTECOQ

Mr Jacques WEBER

Mme Marie-Laure WELTER
$\mathrm{HCN}$ Pédiatrie

$\mathrm{HCN}$ Chirurgie digestive

HCN Thérapeutique

$\mathrm{HCN}$ Pneumologie

HB Pharmacologie

CB Hématologie et transfusion

HCN Médecine Légale

HCN Chirurgie Maxillo-Faciale

HCN Chirurgie digestive

HCN Pédiatrie génétique

$\mathrm{HCN}$ Anesthésiologie - Réanimation chirurgicale

CB Biophysique et traitement de l'image

HB Service Santé Réadaptation

HCN Gynécologie obstétrique

HB Rhumatologie

HCN Physiologie

HCN Physiologie

\section{MAITRES DE CONFERENCES DES UNIVERSITES - PRATICIENS HOSPITALIERS}

$\begin{array}{lll}\text { Mme Noëlle BARBIER-FREBOURG } & \text { HCN } & \text { Bactériologie - Virologie } \\ \text { Mme Carole BRASSE LAGNEL } & \text { HCN } & \text { Biochimie } \\ \text { Mme Valérie BRIDOUX HUYBRECHTS HCN } & \text { Chirurgie Vasculaire } \\ \text { Mr Gérard BUCHONNET } & \text { HCN } & \text { Hématologie } \\ \text { Mme Mireille CASTANET } & \text { HCN } & \text { Pédiatrie } \\ \text { Mme Nathalie CHASTAN } & \text { HCN } & \text { Neurophysiologie } \\ \text { Mme Sophie CLAEYSSENS } & \text { HCN } & \text { Biochimie et biologie moléculaire } \\ \text { Mr Moïse COEFFIER } & \text { HCN } & \text { Nutrition } \\ \text { Mr Serge JACQUOT } & \text { UFR } & \text { Immunologie } \\ \text { Mr Joël LADNER } & \text { HCN } & \text { Epidémiologie, économie de la santé } \\ \text { Mr Jean-Baptiste LATOUCHE } & \text { UFR } & \text { Biologie cellulaire } \\ \text { Mr Thomas MOUREZ } & \text { HCN } & \text { Virologie }\end{array}$


Mr Gaël NICOLAS

$\mathrm{HCN}$ Génétique

Mme Muriel QUILLARD

$\mathrm{HCN}$ Biochimie et biologie moléculaire

Mme Laëtitia ROLLIN

HCN Médecine du Travail

Mr Mathieu SALAUN

HCN Pneumologie

Mme Pascale SAUGIER-VEBER

HCN Génétique

Mme Anne-Claire TOBENAS-DUJARDIN

HCN Anatomie

Mr David WALLON

$\mathrm{HCN}$ Neurologie

\section{PROFESSEUR AGREGE OU CERTIFIE}

Mr Thierry WABLE

UFR Communication

Mme Mélanie AUVRAY-HAMEL

UFR Anglais 


\section{II - PHARMACIE}

\section{PROFESSEURS}

Mr Thierry BESSON

Mr Roland CAPRON (PU-PH)

Mr Jean COSTENTIN (Professeur émérite)

Mme Isabelle DUBUS

Mr François ESTOUR

Mr Loïc FAVENNEC (PU-PH)

Mr Jean Pierre GOULLE(Professeur émérite)

Mr Michel GUERBET

Mme Isabelle LEROUX - NICOLLET

Mme Christelle MONTEIL

Mme Martine PESTEL-CARON (PU-PH)

Mr Rémi VARIN (PU-PH)

Mr Jean-Marie VAUGEOIS

Mr Philippe VERITE

\section{MAITRES DE CONFERENCES}

Mme Cécile BARBOT

Mr Jérémy BELLIEN(MCU-PH)

Mr Frédéric BOUNOURE

Mr Abdeslam CHAGRAOUI

Mme Camille CHARBONNIER (LE CLEZIO)

Mme Elizabeth CHOSSON

Mme Marie Catherine CONCE-CHEMTOB

Mme Cécile CORBIERE
Chimie Thérapeutique

Biophysique

Pharmacologie

Biochimie

Chimie Organique

Parasitologie

Toxicologie

Toxicologie

Physiologie

Toxicologie

Microbiologie

Pharmacie clinique

Pharmacologie

Chimie analytique
Chimie Générale et Minérale

Pharmacologie

Pharmacie Galénique

Physiologie

Statistiques

Botanique

Législation pharmaceutique et économie de la santé

Biochimie 
Mr Eric DITTMAR

Mme Nathalie DOURMAP

Mme Isabelle DUBUC

Mme Dominique DUTERTE- BOUCHER

Mr Abdelhakim ELOMRI

Mr Gilles GARGALA (MCU-PH)

Mme Nejla EL GHARBI-HAMZA

Mme Marie-Laure GROULT

Mr Hervé HUE

Mme Laetitia LE GOFF

Mme Hong LU

M Jérémie MARTINET (MCU-PH)

Mme Marine MALLETER

Mme Sabine MENAGER

Mme Tiphaine ROGEZ-FLORENT

Mr Mohamed SKIBA

Mme Malika SKIBA

Mme Christine THARASSE

Mr Frédéric ZIEGLER

\section{PROFESSEURS ASSOCIES}

Mme Cécile GUERARD-DETUNCQ

Mr Jean-François HOUIVET

\section{PROFESSEUR CERTIFIE}

Mme Mathilde GUERIN

\section{ASSISTANT HOSPITALO-UNIVERSITAIRE}

Mme Anaïs SOARES
Biophysique

Pharmacologie

Pharmacologie

Pharmacologie

Pharmacognosie

Parasitologie

Chimie analytique

Botanique

Biophysique et mathématiques

Parasitologie - Immunologie

Biologie

Immunologie

Toxicologie

Chimie organique

Chimie analytique

Pharmacie galénique

Pharmacie galénique

Chimie thérapeutique

Biochimie

Pharmacie officinale

Pharmacie officinale

Anglais

Bactériologie 
ATTACHES TEMPORAIRES D'ENSEIGNEMENT ET DE RECHERCHE

Mr Souleymane ABDOUL-AZIZE

Biochimie

Mme Hanane GASMI

Galénique

Mme Caroline LAUGEL

Chimie organique

Mr Romy RAZAKANDRAINIBE

Parasitologie 


\section{LISTE DES RESPONSABLES DES DISCIPLINES PHARMACEUTIQUES}

Mme Cécile BARBOT

Mr Thierry BESSON

Mr Roland CAPRON

Mme Marie-Catherine CONCE-CHEMTOB

Mme Elisabeth CHOSSON

Mme Isabelle DUBUS

Mr Abdelhakim ELMORI

Mr Loïc FAVENNEC

Mr Michel GUERBET

Mr François ESTOUR

Mme Isabelle LEROUX-NICOLLET

Mme Martine PESTEL-CARON

Mr Mohamed SKIBA

Mr Rémi VARIN

M. Jean-Marie VAUGEOIS

Mr Philippe VERITE
Chimie Générale et minérale

Chimie thérapeutique

Biophysique

Législation et économie de la santé

Botanique

Biochimie

Pharmacognosie

Parasitologie

Toxicologie

Chimie organique

Physiologie

Microbiologie

Pharmacie galénique

Pharmacie clinique

Pharmacologie

Chimie analytique 


\section{III - MEDECINE GENERALE}

\section{PROFESSEUR}

Mr Jean-Loup HERMIL (PU-MG) UFR Médecine générale

\section{MAITRE DE CONFERENCE MEDECINE GENERALE}

Mr Matthieu SCHUIERS (MCU-MG) UFR Médecine générale

\section{PROFESSEURS ASSOCIES A MI-TEMPS}

Mr Emmanuel LEFEBVRE

Mme Elisabeth MAUVIARD

Mr Philippe NGUYEN THANH

Mme Marie Thérèse THUEUX
UFR Médecine générale

UFR Médecine générale

UFR Médecine générale

UFR Médecine générale

\section{MAITRE DE CONFERENCES ASSOCIE A MI-TEMPS}

MrPascal BOULET

Mr Emmanuel HAZARD

Mme Marianne LAINE

Mme Lucile PELLERIN

Mme Yveline SEVRIN
UFR Médecine générale

UFR Médecine générale

UFR Médecine générale

UFR Médecine générale

UFR Médecine générale 


\section{ENSEIGNANTS MONO-APPARTENANTS}

\section{PROFESSEURS}

Mr Serguei FETISSOV (med)

Physiologie (ADEN)

Mr Paul MULDER (phar)

Sciences du Médicament

Mme Su RUAN(med)

Génie Informatique

\section{MAITRES DE CONFERENCES}

Mr Sahil ADRIOUCH(med)

Biochimie et biologie moléculaire(Unité Inserm 905)

Mme Gaëlle BOUGEARD-DENOYELLE (med) Biochimie et biologie moléculaire(UMR 1079)

Mme Carine CLEREN (med)

Neurosciences (Néovasc)

M. Sylvain FRAINEAU(phar)

Physiologie (Inserm U 1096)

Mme Pascaline GAILDRAT (med)

Génétique moléculaire humaine (UMR 1079)

Mr Nicolas GUEROUT (med)

Chirurgie Expérimentale

Mme Rachel LETELLIER(med)

Physiologie

Mme Christine RONDANINO(med)

Physiologie de la reproduction

Mr Antoine OUVRARD-PASCAUD (med)

Physiologie (Unité Inserm 1076)

Mr Frédéric PASQUET

Sciences du langage, orthophonie

Mme Isabelle TOURNIER (med)

Biochimie (UMR 1079)

CHEF DES SERVICES ADMINISTRATIFS : Mme Véronique DELAFONTAINE

HCN - Hôpital Charles Nicolle

CB - Centre Henri Becquerel

CRMPR - Centre Régional de Médecine Physique et de Réadaptation
HB - Hôpital de BOIS GUILLAUME

CHS - Centre Hospitalier Spécialisé du Rouvray

SJ - Saint Julien Rouen 


\section{Table des matières}

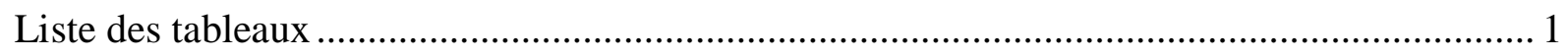

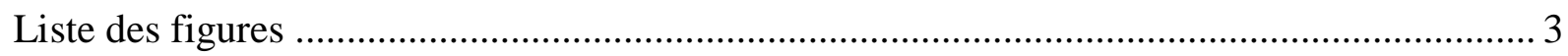

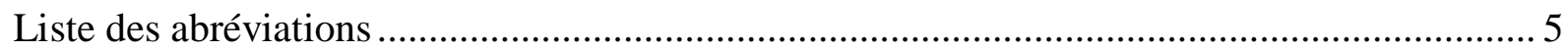

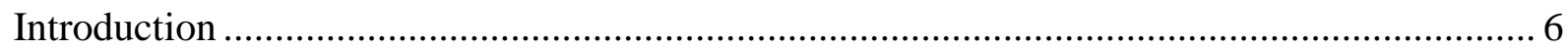

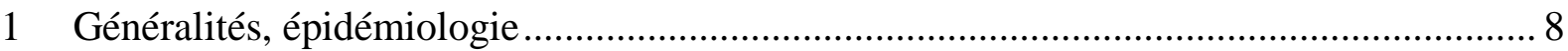

1.1 Médicaments psychoactifs ou à risque pour la conduite .......................................... 8

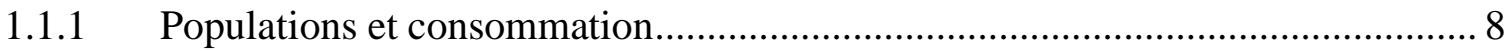

1.1.2 Législation, pictogrammes et moyens d'informations ................................... 10

1.1.3 Médicaments et substances concernés ......................................................... 15

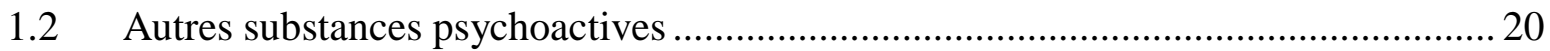

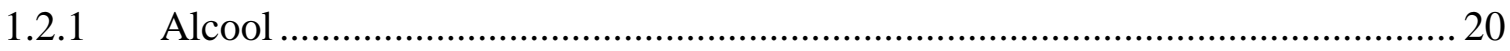

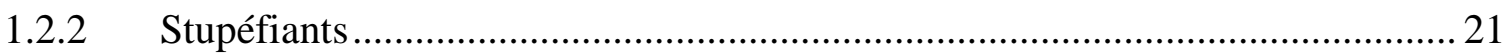

1.2.3 Usage détourné de médicaments psychoactifs ............................................. 25

1.3 Accidents de la voie publique liés à la consommation de produits psychoactifs en France 27

2 Travaux Personnels : étude rétrospective d'une série de cas au CHU de Rouen.............. 31

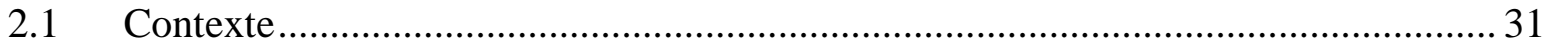

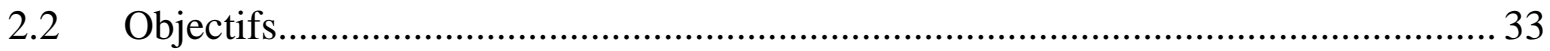

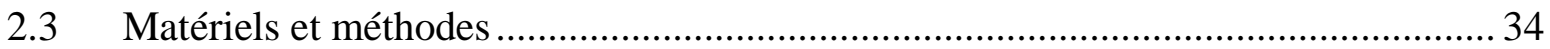

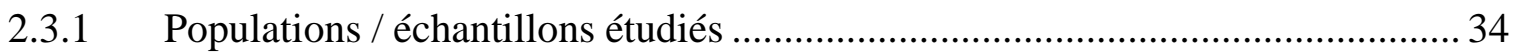




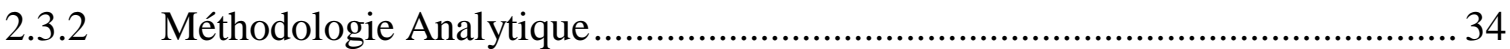

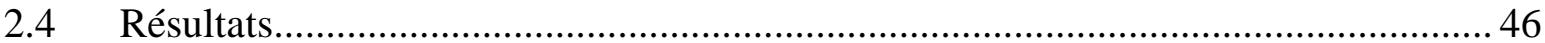

2.4.1 Caractéristiques démographiques des échantillons étudiés.............................. 46

2.4.2 Estimation de l'incidence des médicaments chez les conducteurs.................... 48

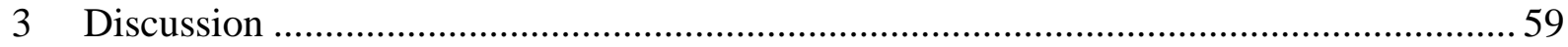

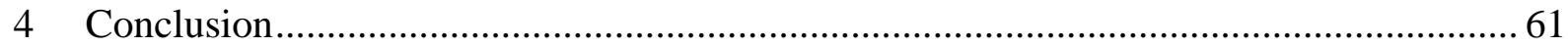

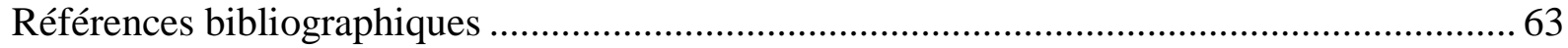




\section{Liste des tableaux}

Tableau I Liste indicative des substances de niveau 1 susceptibles d'altérer la capacité de la conduite automobile ou d'engins professionnels

Tableau II Liste indicative des substances de niveau 2 susceptibles d'altérer la capacité de la conduite automobile ou d'engins professionnels

Tableau III Liste indicative des substances de niveau 3 susceptibles d'altérer la capacité de la conduite automobile ou d'engins professionnels

Tableau IV Liste des molécules d'intérêts recherchées par screening avec leurs Temps de rétention (Tr) et un niveau de vigilance non défini

Tableau V Liste des molécules d'intérêts recherchées par screening avec leurs Temps de rétention $(\mathrm{Tr})$ et un niveau de vigilance 1

Tableau VI Liste des molécules d'intérêts recherchées par screening avec leurs Temps de rétention (Tr) et un niveau de vigilance 2

Tableau VII Liste des molécules d'intérêts recherchées par screening avec leurs Temps de rétntion $(\mathrm{Tr})$ et un niveau de vigilance 3

Tableau VIII Répartition des individus de la population étudiée. 46

Tableau IX Répartition de la population étudiée par sexe . .46

Tableau X Répartition des cas positifs et négatifs THC en fonction du sexe 50

Tableau XI Répartition positif/négatif stupéfiants en fonction du sexe.

Tableau XII Répartition des cas positifs et négatifs en médicaments en fonction du sexe. 52

Tableau XIII Fréquence et molécules de niveau non défini retrouvées chez les conducteurs étudiés. 
Tableau XIV Fréquence et molécules de niveau de vigilance 1 retrouvées chez les conducteurs étudiés.

Tableau XV Fréquence et molécules de niveau de vigilance 2 retrouvées chez les conducteurs étudiés.

Tableau XVI Fréquence et molécules de niveau de vigilance 3 retrouvées chez les conducteurs étudiés.

Tableau XVII Tableau récapitulatif de la fréquence des scores (stupéfiant + alcool + médicaments) obtenus sur l'échantillon

Tableau XVIII Tableau récapitulatif de la fréquence des scores (association de médicaments uniquement) obtenus chez les conducteurs étudiés. 55 


\section{Liste des figures}

Figure 1 : Evolution des ventes de spécialités pharmaceutiques aux officines, en nombre de boîtes 8

Figure 2 : Pictogramme unique utilisé jusqu'en 1999 (source: document AFSSAPS). 10

Figure 3 : Pictogramme Niveau 1 (source: document AFSSAPS) : risque modéré. 11

Figure 4 : Pictogramme Niveau 2 (source: document AFSSAPS) : risque relativement important. 11

Figure 5 : Pictogramme Niveau 3 (source: document AFSSAPS) : risque important. 12

Figure 6 : Exemple d'affiche de prévention: conduite et médicament (11). 13

Figure 7 : Extrait de brochure « conseils » destinée au patient (12). 14

Figure 8 : Evolution entre 1992 et 2014 de la proportion des consommateurs de cannabis en france, au cours de l'année parmi les 18-64 ans par sexe (en \%). (22) 22

Figure 9 : Evolution de l'usage du cannabis entre 2010 et 2014 selon l'âge et le sexe (\%)(22). 23

Figure 10 : Evolution de l'usage dans l'année des principales autres drogues illicites entre 1992 et 2014, parmi les 18-64 ans (en \%)(22). 24

Figure 11 : Extrait du tableau ANSM sur la surveillance des médicaments psychoactifs, ici le tramadol(26). 26

Figure 12 : Facteurs comportementaux chez les auteurs présumés d'accidents mortels de septembre 2014 à décembre 2017 (26). 28

Figure 13 : Facteurs comportementaux chez les auteurs présumés d'accidents mortels sur routes bidirectionnelles hors agglomérations en 2017 (26). 28

Figure 14 : Facteurs comportementaux chez les auteurs présumés d'accidents mortels en agglomérations (26).

Figure 15 : Représentation schématique d'un système HPLC (32). 36 
Figure 16 : Photographie du système HPLC-MS/MS utilisé au cours de ce travail. 38

Figure 17 : Tracé caractéristique d'un échantillon analysé contenant du citalopram (niveau de vigilance pour a conduite : 2 ). 45

Figure 18 : Répartition des Procès Verbaux (PV) en fonction de leur origine géographique.. 47 Figure 19 : Répartition des individus de l'échantillon en fonction de la présence ou non d'éthanol. 49

Figure 20 : Répartition des individus de l'échantillon en fonction de la présence ou non de THC.

Figure 21 : Répartition des individus étudiés en fonction de la présence ou non de stupéfiants.

Figure 22 : Répartition des individus étudiés en fonction de la présence ou non de médicaments.

Figure 23 : Histogramme représentant les molécules et leur fréquence chez les conducteurs étudiés. 57

Figure 24 : Graphique représentant la répartition des médicaments chez les individus étudiés. 


\section{Liste des abréviations}

AFSSAPS : Agence Française de la Sécurité Sanitaire des Produits de Santé ANPAA : Association Nationale de Prévention en Alcoologie et Addictologie ANSM : Agence Nationale de sécurité du médicament et des produits de santé ATC: Anatomical Therapeutic Chemical Classification System

AVP: Accident de la Voie Publique

CHU : Centre Hospitalo-Universitaire

CEIP: Centre d'Evaluation et d'Information sur la Pharmacodépendance

CPG:Chromatographie en Phase Gazeuse

HPLC:Chromatographie Liquide Haute Performance

MDT : Médicament

NPS: Nouveaux Produits de Synthèse

OCDE : Organisation de Coopération et de Développement Economiques

OFDT : Observatoire Français des Drogues et des Toxicomanies

PV : Procès-Verbal

SNC : Système Nerveux Central

SNP : Système Nerveux Périphérique

ST : Sang Total

THC : Tétrahydrocannabinol 


\section{Introduction}

La sécurité routière est un enjeu majeur de la société actuelle. L'objectif de la lutte contre l'insécurité routière est notamment de faire diminuer le nombre d'accidents et de morts sur les routes françaises, qui était évalué en 2017 à 61570 accidents (métropole et outre-mer confondues) dont environ 3700 décès, ce qui représente une augmentation d'accidents par rapport à $2016(+3 \%)$. Dans la catégorie des automobilistes, bien que l'on constate une légère baisse de la mortalité chez les moins de 25 ans (20 tués de moins par rapport à 2016), il est observé une augmentation de la mortalité chez des séniors de 65 ans et plus (50 tués de plus entre 2016 et 2017)(1).Dans cette lutte contre l'insécurité routière, plusieurs méthodes sont utilisées sur le terrain par les forces de l'ordre : le contrôle de la vitesse, l'information et la mise en place de contrôles des gestes essentiels de sécurité routière (ceinture, fatigue, téléphone au volant, ...), la prévention et/ou la répression envers les consommations d'alcool et/ou de stupéfiants qui restent avec la vitesse les premières causes de la mortalité routière.

Afin de détecter l'usage au volant de substances psychotropes chez les automobilistes, les forces de l'ordre peuvent procéder à différents dépistages : tests salivaires (depuis fin 2017 : recherche des principaux produits stupéfiants tels que le cannabis, la cocaïne, les opiacés et les dérivés amphétaminiques comme l'ecstasy = MDMA) et dépistage dans le sang de ces stupéfiants ou de l'alcool éthylique(alcoolémie)(2). Ces analyses pour objet de manifester la vérité par un tribunal civil ou pénal et de préparer certaines dispositions légales, réglementaires ou administratives (3). Elles sont réalisées conformément aux dispositions légales, dans un laboratoire spécialisé, selon des méthodologies reconnues spécifiques et sensibles, sous la responsabilité d'experts de justice. 
Malgré ces mesures de lutte mises en œuvre contre la consommation d'alcool ou de stupéfiants sur les routes, l'impact de certains médicaments n'est pas évalué en pratique courante. En effet, il est important de rappeler que certains médicaments, qu'ils soient psychoactifs ou non, peuvent avoir un impact sur la vigilance au volant et ce, d'autant plus si leur prise est combinée avec celle d'alcool et/ou de stupéfiants.

Dans ce contexte, le conseil pharmaceutique apparait très important lors de la dispensation de médicaments susceptibles d'influencer la capacité à la conduite automobile.

L'objectif principal de ce travail de thèse était d'évaluer l'incidence de la consommation de médicaments « à risque » dans une population de conducteurs impliqués dans des contrôles routiers, dans des accidents ou des délits de la circulation. Nous établissons dans une première partie de ce travail un état des lieux de l'implication de la consommation de médicaments, de l'alcool et de stupéfiants en France dans les accidents / délits routiers. Nous développons également les moyens de dépistage, les méthodologies associées, ainsi que leurs limites et avantages. Enfin, nous présentons les résultats d'une étude menée à partir d'une série de cas suivis entre 2016 et 2017 dans le Laboratoire de Pharmacologie-Toxicologie du CHU de Rouen. 


\section{Généralités, épidémiologie}

\subsection{Médicaments psychoactifs ou à risque pour la conduite}

\subsubsection{Populations et consommation}

L'Agence Nationale de Sécurité du Médicament et des produits de santé (ANSM) publie régulièrement des rapports sur la consommation et les ventes des médicaments en France. Le dernier datant de juin 2014, mais traitant de l'année 2013, indique qu'en France 2800 substances actives différentes étaient commercialisées, soit 11000 spécialités. En officine, les formes orales sont quantitativement les plus vendues (environ $2 / 3$ du marché), contrairement au domaine hospitalier où les formes injectables sont prédominantes. D'un point de vue économique les analgésiques sont les plus vendus, suivis des antidiabétiques et des antiasthmatiques. Quantitativement, on distingue principalement les analgésiques, puis les psycholeptiques et enfin les antibiotiques. En 2013, on comptabilisait environ 3,1 milliards de boîtes consommées, soit en moyenne 48 boites / habitant / an (4).

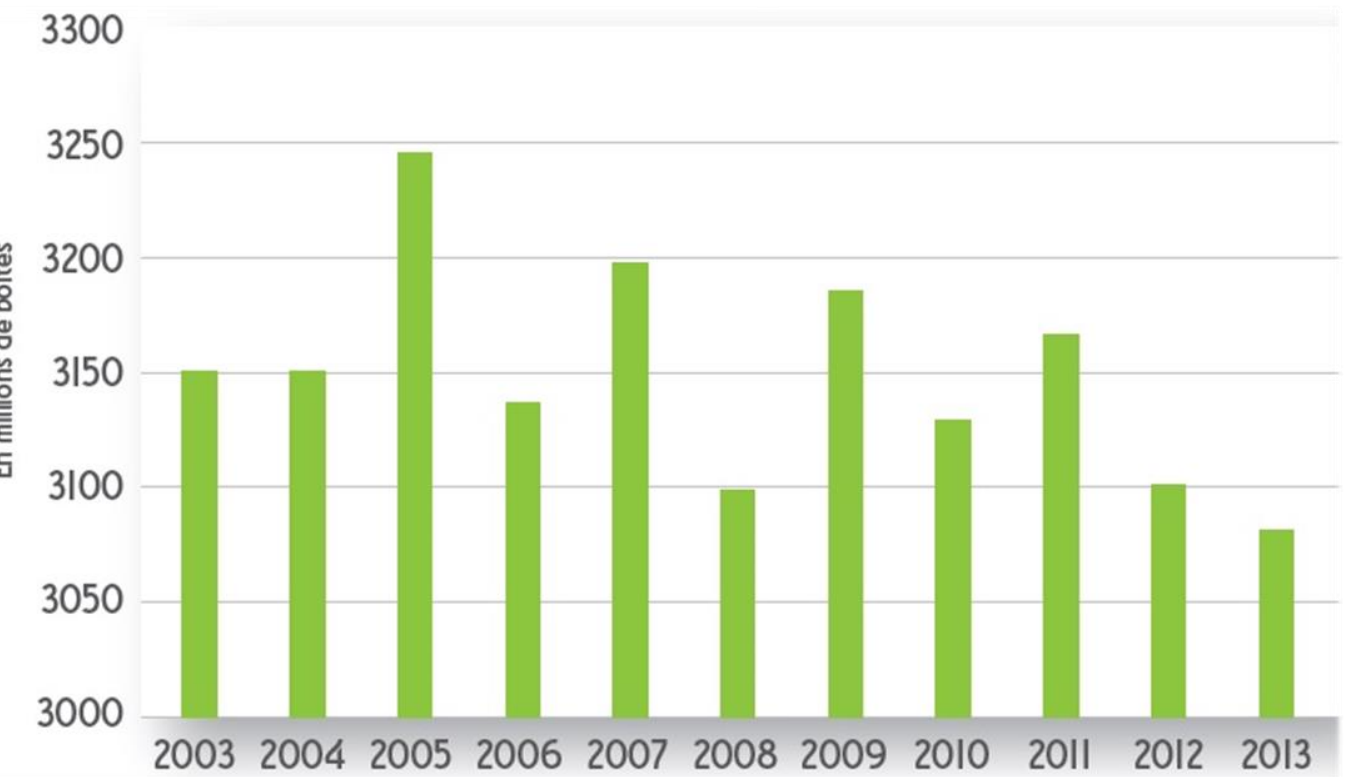

FIGURE 1 : EVOLUTION DES VENTES DE SPECIALITES PHARMACEUTIQUES AUX OFFICINES, EN NOMBRE DE BOITES 
D'après le graphique de l'ANSM présenté en figure 1, on peut néanmoins constater une tendance à la diminution de la consommation des médicaments en France depuis plusieurs années (4). En revanche, les données recueillies par l'OCDE en 2015montraientune augmentation de manière générale de la consommation pharmaceutique dans les pays membres (Allemagne Australie, Autriche, Belgique, Canada, Chili, Corée du Sud, Danemark, Espagne, Estonie, États-Unis, Finlande, France, Grèce, Hongrie, Irlande, Islande, Israël, Italie, Japon, Luxembourg, Mexique, Norvège, Nouvelle-Zélande, Pays-Bas, Pologne, Portugal, République tchèque, Royaume-Uni, Slovaquie, Slovénie, Suède, Suisse, Turquie), par les médicaments des pathologies liées à l'âge et les pathologies chroniques, comme par exemple : les antihypertenseurs (x2 entre 2000 et 2013), les hypocholestérolémiants (x3), les antidiabétiques (x2) et les antidépresseurs (très variable selon le pays, en France et en Angleterre, on constate une augmentation de la consommation liée à l'allongement de la prise en charge). Chacune de ces classes thérapeutiques pourrait présenter un impact négatif sur la conduite automobile ou la conduite d'engins dans un contexte professionnel par leur action directe (par ex : SNC), ou par leurs effets secondaires (hypotension, hypoglycémie, etc.)(5).

Avec une espérance de vie en augmentation, ce sont les personnes âgées, souvent atteintes de polypathologies, qui représentent la tranche de la population la plus consommatrice de spécialités pharmaceutiques. Néanmoins, la population de jeunes individus n'est pas épargnée pour autant. En effet, selon l'Observatoire Français des Drogues et des Toxicomanies (OFDT), chez les jeunes, comme chez les adultes, les femmes utilisent d'avantage de médicaments psychotropes du fait de leur plus forte consommation de soins par rapport aux hommes. 


\subsubsection{Législation, pictogrammes et moyens d'informations}

Comme précisé dans le Code de la Santé Publique (CSP), les laboratoires pharmaceutiques commercialisant des produits de santé sont tenus de faire figurer un pictogramme informatif concernant le potentiel impact de la molécule sur la conduite d'engins (automobiles, engins professionnels, ...). D'après le deuxième alinéa de l'article R. 5121-139 du code de la santé publique : «Lorsque le médicament ou produit a des effets sur la capacité de conduire des véhicules ou d'utiliser des machines, mentionnés dans le résumé des caractéristiques du produit, son conditionnement extérieur comporte un pictogramme, dont le modèle est déterminé, après avis du directeur général de l'Agence Nationale de Sécurité du Médicament et des produits de santé, par arrêté du ministre chargé de la santé »(6).

Le pictogramme se décline en trois modèles en fonction du niveau de risque potentiel de la substance ou de ses effets sur la capacité à conduire des véhicules. Jusqu'en 1999, un seul type de pictogramme (figure 2) figurait sur les emballages secondaires des médicaments.

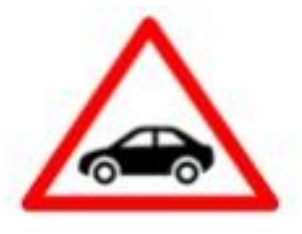

FIGURE 2 : PICTOGRAMME UNIQUE UTILISE JUSQU'EN 1999 (SOURCE: DOCUMENT AFSSAPS).

A partir de 2005 et dans le but d'une harmonisation européenne, l'AFSSAPS a mis en place 3 nouveaux pictogrammes, comportant un niveau de risque $(1,2,3)$, en fonction des potentiels effets de la substance sur la conduite, associant une couleur différente en fonction du niveau (respectivement: jaune, orange, rouge) à une explication sur la conduite à tenir. Les 
médicaments concernés peuvent être soumis à prescription ou non, en libre accès ou non. Les médicaments peuvent avoir un impact sur la conduite de véhicules en altérant les capacités de jugement, de discernement, en diminuant les réflexes, en gênant la vue, en donnant des vertiges ou en perturbant les mouvements.

Les figures 3 à 5 présentent les 3 différents pictogrammes proposés actuellement.

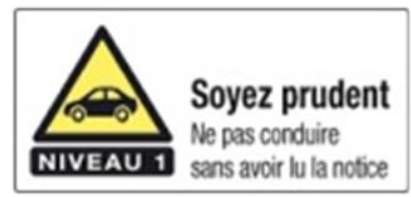

FIgURE 3 : PICTOGRAMmE NiVEAU 1 (SOURCE: DOCUMENT AFSSAPS) : RISQUE MODERE.

Pour les substances de niveau 1, la conduite automobile n'est pas contre-indiquée, mais le patient doit être informé des potentiels effets (médicaments contre le rhume : somnolence ; antihypertenseurs : vertiges, etc.).

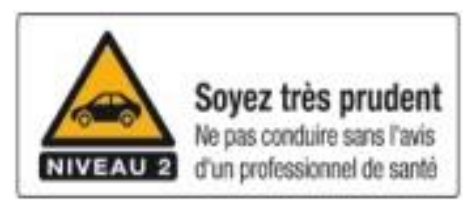

Figure 4 : Pictogramme NiveAu 2 (SOURCE: DoCUment AFSSAPS) : RISQUE RELATIVEMENT IMPORTANT 
Pour les substances de niveau 2, la conduite d'engins n'est pas une contre-indication absolue mais elle peut être remise en cause. Néanmoins, il revient au patient d'en discuter avec un professionnel de santé, le médecin prescripteur ou le pharmacien dispensant le médicament, afin d'évaluer individuellement le risque associé. On retrouve dans cette catégorie des médicaments antidépresseurs, des antalgiques (codéine, morphine...), des antidiabétiques, des antiépileptiques, des antiparkinsoniens, mais aussi des antiallergiques, et des molécules indiquées dans le mal des transports.

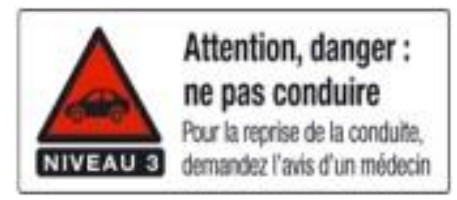

FIGURE 5 : PICTOGRAMmE NIVEAU 3 (SOURCE: DOCUMENT AFSSAPS) : RISQUE IMPORTANT.

Pour les substances de niveau 3, l'aptitude à la conduite automobile est remise en cause le temps de leur utilisation. Ce sont ces médicaments qui présentent le risque le plus élevé, entrainant une incapacité temporaire rendant impossible la conduite d'automobiles ou de certains engins professionnels. On retrouve dans cette catégorie presque tous les somnifères, les tranquillisants et neuroleptiques, les anesthésiques généraux et certains collyres dilatant la pupille ou susceptibles de perturber la vue. Le médecin et/ou le pharmacien sont théoriquement tenus d'informer le patient sur le délai à respecter avant de pouvoir reprendre le volant (7)(8).

D'après une étude française sur l'impact des pictogrammes et la corrélation avec les accidents de la route, le risque était accru chez les sujets exposés aux benzodiazépines et aux hypnotiques avant l'implantation de ce dispositif en 2005. Puis à la mise en place des 
pictogrammes, le risque diminue significativement (de 2005 à 2008/2009), mais une réaugmentation du risque d'accident apparait après 2008/2009 (9).

C'est pourquoi la prévention auprès des professionnels de santé apparaît importante, comme par exemple via les bulletins diffusés par les services de pharmacovigilance, comme au CHU de Rouen récemment où il est rappelé les trois pictogrammes existants, un rappel épidémiologique (3,4\% des accidents mortels de la route sont liés aux médicaments, 11 millions de personnes consomment des benzodiazépines en France), un lien vers la liste des substances ayant un pictogramme, ainsi que les mises à jour des listes (10).

La prévention par information largement diffusée dans la population générale joue également un rôle important. En collaboration avec l'ordre des pharmaciens, le Cespharm, l'ANSM et la sécurité routière, des affiches ont été créées afin de sensibiliser les patients sur le risque potentiel lié aux médicaments, pour qu'ils s'informent auprès d'un professionnel de santé et qu'ils prennent connaissance des risques potentiels via la notice de leur traitement (figures 6 et 7).

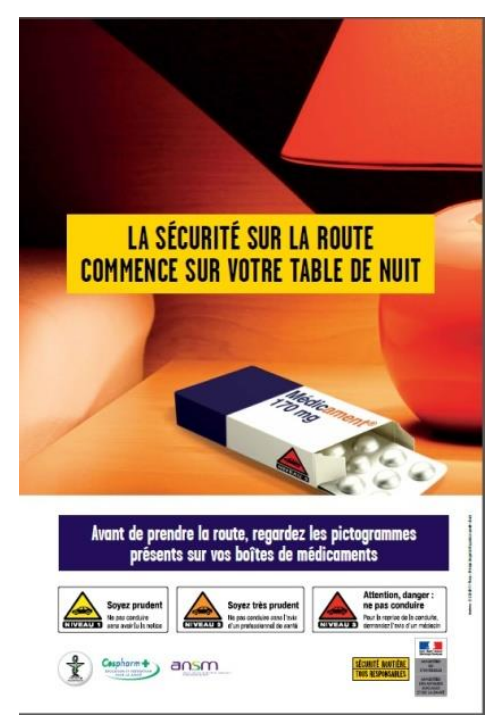




\section{Afin de limiter les risques pour la conduite, respectez les heures et les conditions de prise de votre médicament. Ne modifiez pas les doses ou niarrêtez pas votre traitement sans l'avis d'un professionnel de santé.}

FiguRE 7 : EXTRAIT DE BROCHURE « CONSEILS » DESTINEE AU PATIENT (12).

D'un point de vue règlementaire, les laboratoires pharmaceutiques sont tenus en cas de risque potentiel de faire figurer le pictogramme approprié sur le conditionnement secondaire comme expliqué précédemment. Les patients ont eux aussi des obligations mais le plus souvent méconnues du grand public. En effet, les patients atteints de certaines pathologies devraient se déclarer auprès de leur préfecture de rattachement afin d'effectuer une consultation médicale destinée à évaluer leur capacité ou non à conduire, c'est-à-dire permettre l'obtention, le maintien du permis de conduire pour une durée limitée, ou dans certains cas l'interdiction de la conduite automobile ou d'engins professionnels (13). Par exemple, un patient diabétique traité par insuline ne s'étant pas déclaré auprès de la préfecture, et qui est responsable d'un accident suite à une hypoglycémie (prouvée) s'expose à un risque de non prise en charge par son assureur. Il apparait donc important pour les professionnels de santé et les patients de prendre connaissance de cette liste de médicaments « à risques »afin de faciliter l'information des patients. 


\subsubsection{Médicaments et substances concernés}

Les molécules présentées ici seront celles dont le mécanisme d'action est susceptible d'agir sur le SNC et/ou SNP, ou dont les effets secondaires peuvent avoir des conséquences sur la conduite automobile. C'est pourquoi, dans certains cas on parlera de substance psychoactive, c'est-à-dire une substance chimique susceptible de moduler l'activité mentale et d'altérer la vigilance du conducteur (14).

D'après l'arrêté du 8 août 2008, modifié le 13 mars 2017, nous présentons dans les Tableaux I à III une liste non exhaustive des substances susceptibles d'entrer dans la composition de médicaments et de produits pouvant altérer la capacité de conduite automobile ou de machines motorisées en fonction de leur niveau de vigilance. Il est possible de retrouver les molécules présentées ci-dessous selon la classification ATC à 5 niveaux, c'est-à-dire selon les classes pharmacologiques et/ou indications sur le site légifrance (8).

TABLEAU I : LISTE INDICATIVE DES SUBSTANCES DE NIVEAU 1 SUSCEPTIBLES D'ALTERER LA CAPACITE DE LA CONDUITE AUTOMOBILE OU D'ENGINS PROFESSIONNELS (8).

Liste alphabétique des substances avec pictogramme de niveau I

\begin{tabular}{c|c|c|c} 
Acéclofénac & Alminoprofène & $\begin{array}{c}\text { Association d'inhibiteurs de } \\
\text { la transcriptase reverse }\end{array}$ & Buséréline \\
\hline $\begin{array}{c}\text { Acide acétylsalicylique } \\
\text { association à la codéine }(<20 \mathrm{mg} \\
\text { codéine base / prise })\end{array}$ & Alprostadil & Atropine en association & Buspirone \\
\hline Acide cromoglicique & Amantadine & $\begin{array}{c}\text { Autres médicaments du } \\
\text { rhume en association (ex. } \\
\text { ibuprofène) }\end{array}$ & Calcitonine de saumon \\
\hline Acide méfénamique & Amlodipine & Azélastine & Candésartan \\
\hline Acide niflumique & Amodiaquine & Bénazépril & Captodiame \\
\hline Acide pipémidique & Anastrozole & Benfluorex & Captopril \\
\hline Acide tiaprofénique & Atg de l'angiotensine II et \\
diurétiques & Bimatoprost & Certéolol \\
\hline Acide tranexamique & Antiméningococcique C & Bosentan & Ceftazidime \\
\hline Acitrétine & Apraclonidine & Brimonidine & Célécoxib \\
\hline Alcaloïdes de l'opium et dérivés & Aprindine & Brinzolamide & $\begin{array}{c}\text { Céthéxonium et } \\
\text { phényltoloxamine }\end{array}$ \\
\hline Alfuzosine & Association (alcaloïdes de \\
\hline- l'opium et dérivés)
\end{tabular}




\begin{tabular}{|c|c|c|c|}
\hline Cétirizine & Flécaïnide & Manidipine & Progestérone \\
\hline Chloroquine & Floctafénine & Méfloquine & $\begin{array}{l}\text { Proguanil en association } \\
\text { à la chloroquine }\end{array}$ \\
\hline Chlortétracycline & Fluorescéine (injectable) & Mélatonine & Propafénone \\
\hline Cibenzoline & Flurbiprofène & Méloxicam & $\begin{array}{l}\text { Pseudoéphédrine en } \\
\text { association }\end{array}$ \\
\hline Cilazapril & Fosinopril & Méropénème & Quinapril \\
\hline Ciprofloxacine & Gentamicine & Métipranolol & Quinine \\
\hline Clomifène & Granisétron & Métoprolol et félodipine & Quinupristine/dalfopristine \\
\hline Codéine & $\begin{array}{l}\text { Hydrocortisone et anti- } \\
\text { infectieux }\end{array}$ & Métronidazole & Rasagiline \\
\hline Cyprotérone & Hydroxyquinidine & Métyrapone & Ribavirine \\
\hline $\begin{array}{l}\text { Dérivés de l'opium et } \\
\text { expectorants }\end{array}$ & Ibuprofène & Mexilétine & Rifamycine \\
\hline Desloratadine & Imidapril & Midodrine & Riluzole \\
\hline Dexaméthasone & Indocyanine & Moexipril & Rimexolone \\
\hline Dexaméthasone et anti-infectieux & Indométacine & Morniflumate & Ritonavir \\
\hline Dexibuprofène & Indométacine et anti-infectieux & Nabumétone & Roxithromycine \\
\hline Dexkétoprofène & $\begin{array}{l}\text { Inhibiteurs de l'enzyme de } \\
\text { conversion et diurétiques }\end{array}$ & Naproxène & Secnidazole \\
\hline Dextrométhorphane & lodixanol & Nimésulide & Sélégiline \\
\hline Diclofénac & Irbésartan & Nitrendipine & Sildénafil \\
\hline Diclofénac en association & Isosorbide dinitrate & Noscapine & $\begin{array}{l}\text { Spiramycine en } \\
\text { association avec d'autres } \\
\text { antibactériens }\end{array}$ \\
\hline Diéthylcarbamazine & Isosorbide mononitrate & $\begin{array}{l}\text { Nystatine en association } \\
\text { (pour les spécialités } \\
\text { contenant du } \\
\text { métronidazole) }\end{array}$ & Sulindac \\
\hline $\begin{array}{c}\text { Dihydroergotamine (pulvérisation } \\
\text { nasale) }\end{array}$ & Isotrétinoïne & Olmésartan medoxomil & Tadalafil \\
\hline Dipivéfrine & Isradipine & Olopatadine & Tamoxifène \\
\hline Disopyramide & Kétoprofène & Ondansétron & Tamsulosine \\
\hline Dolasétron & Kétotifène & Ornidazole & Teicoplanine \\
\hline Dorzolamide & Latanoprost & Oxytétracycline & Telmisartan \\
\hline Doxazosine & Létrozole & Papavérine & Ténoxicam \\
\hline Enalapril & Leuproréline & $\begin{array}{c}\text { Paracétamol en association } \\
\text { à la codéine ou à l'opium }(< \\
20 \mathrm{mg} \text { codéine base par } \\
\text { prise) }\end{array}$ & Térazosine \\
\hline $\begin{array}{l}\text { Encéphalite transmise par les } \\
\text { tiques, virus entier inactivé }\end{array}$ & Lévobunolol & Parécoxib & Tériparatide \\
\hline Eprosartan & Lévocétirizine & Pentaérithrityl tétranitrate & Timolol \\
\hline Ergotamine en association & Lidocaïne & Pentoxyvérine & Timolol en association \\
\hline Ertapénem & Lisinopril & Périndopril & Tinidazole \\
\hline Ethylmorphine & Lopéramide & Phénylbutazone & Tobramycine \\
\hline Etifoxine & Lopéramide en association & Pholcodine & Toniques \\
\hline Etodolac & Lopéramide oxyde & Piroxicam & Trandolapril \\
\hline Exemestane & Loratadine & Pralidoxime & $\begin{array}{l}\text { Trandolapril et inhibiteurs } \\
\text { calciques }\end{array}$ \\
\hline Félodipine & Loracarbef & Praziquantel & Travoprost \\
\hline Fénoprofène & Loratadine en association & Prazosine & Triclabendazole \\
\hline
\end{tabular}




\begin{tabular}{c|c|c|c} 
Trinitrine & Tropisétron & Valsartan & Varénicline \\
\hline Triptoréline & Urapidil & Vardénafil & \\
\hline
\end{tabular}

TABLEAU II : LISTE INDICATIVE DES SUBSTANCES DE NIVEAU 2 SUSCEPTIBLES D'ALTERER LA CAPACITE DE LA CONDUITE AUTOMOBILE OU D’ENGINS PROFESSIONNELS(8).

Liste alphabétique des substances avec pictogramme de niveau II

\begin{tabular}{|c|c|c|c|}
\hline Acétylcholine & Capécitabine & Déféroxamine & Flupentixol (per os) \\
\hline $\begin{array}{l}\text { Acétylsalicylate de lysine }+ \\
\text { métoclopramide }\end{array}$ & Carbachol & Désipramine & Fluphénazine (per os) \\
\hline Acide nalidixique & Carbamazépine & Dexchlorphéniramine & Fluvoxamine \\
\hline Acide pamidronique & Carbinoxamine & Dextromoramide & Fomépizole \\
\hline Acide valproïque & Carbutamide & Diazépam (per os) & Fosphénytoïne \\
\hline Adesleukine & Carpipramine & Dihexyvérine & Frovatriptan \\
\hline Alemtuzumab & Chlophénoxamine & Dihydrocodéine & Gabapentine \\
\hline Alimémazine & Chlorphénamine & Diménhydrinate & Galantamine \\
\hline Almotriptan & Chlorpromazine (per os) & Diphénhydramine & Ganciclovir \\
\hline Amikacine & Ciprofloxacine & Disulfirame & Gemcitabine \\
\hline Amisulpride (per os) & Cisplatine & Donépézil & Gentamicine \\
\hline Amitriptyline & Citalopram & Dosulépine & Glibenclamide \\
\hline Amoxapine & Cladribine & Doxépine & Glibornuride \\
\hline Antitussifs et expectorants & $\begin{array}{l}\text { Clidinium en association } \\
\text { avec des psycholeptiques }\end{array}$ & Doxorubicine & Gliclazide \\
\hline Antitussifs et mucolytiques & Clobazam & Efavirenz & Glimépiride \\
\hline Apomorphine & Clofazimine & Elétriptan & Glipizide \\
\hline Articaïne & Clomipramine & Enoxacine & Guanfacine \\
\hline Atropine & Clonazépam (per os) & Entacapone & Halopéridol (per os) \\
\hline Baclofène & Clonidine & Escitalopram & Halopéridol en association \\
\hline Bexarotène & Clorazépate (per os) & Ethambutol & Hydromorphine \\
\hline Bipéridène & Clotuazépam & $\begin{array}{c}\text { Ethambutol + isoniazide + } \\
\text { pyrazinamide + rifampicine }\end{array}$ & Hydroxychloroquine \\
\hline Bortezomid & Clozapine & Ethambutol + Isoniazide & Hydroxyzine \\
\hline Bromocriptine & Codéine en association & Ethosuximide & Ifosfamide \\
\hline Bromphéramine & Cyamémazine (per os) & Etoposide & Imatinib \\
\hline Buclizine & Cyproheptadine & Felbamate & Imipramine \\
\hline Bupivacaïne en association & Cytarabine & Fentanyl (TD) & Insuline asparte \\
\hline Buprénorphine & Dacarbazine & Fludarabine & Insuline détémir \\
\hline Bupropion & Dactinomycine & Flumarizine & Insuline glargine \\
\hline Butylscopolamine & Dantrolène & Fluméquine & Insuline glulisine \\
\hline Cabergoline & Daunorubicine & Flunisaone (per os) & Insuline humaine \\
\hline Cabergoline & Déférasirox & Fluoxétine & Insuline lispro \\
\hline
\end{tabular}




\begin{tabular}{|c|c|c|c|}
\hline Interféron & Morphine en association & Piracétam & Topiramate \\
\hline Iproniazide & Moxifloxacine & Piribédil & Toxine botulique \\
\hline Irinotécan & Moxonidine & Pizotifène & Tramadol \\
\hline Isothipendyl & Nalbuphine & Posaconazole & Trétinoïne \\
\hline Ivabradine & Naltrexone & Pramipexole & Trifluopérazine \\
\hline Kétotifène & Naphazoline en association & Prégabaline & Trihéxyphénidyle \\
\hline Lamotrigine & Naratriptan & Primidone & Trimipramine \\
\hline Lémalidomide & Natéglinide & Procaïne & Tripolidine \\
\hline Lévétiracétam & Néfopam & Progabide & Tropatépine \\
\hline $\begin{array}{l}\text { Lévodopa + inhibiteur de la } \\
\text { catéchol-O-méthyltransférase }\end{array}$ & Nétilmicine & $\begin{array}{l}\text { Prométhazine en } \\
\text { association }\end{array}$ & Trospium \\
\hline $\begin{array}{c}\text { Lévodopa }+ \text { inhibiteur de la dopa } \\
\text { décarboxylase }\end{array}$ & Nilutamide & Quinagolide & Valganciclovir \\
\hline Lévofloxacine & Norfloxacine & Raltitrexed & Valpromide \\
\hline Lévomépromazine (per os) & Ofloxacine & Répaglinide & Venlafaxine \\
\hline Lidocaïne & Olanzapine (per os) & Rilménidine & Véralipride \\
\hline Linézolide & Opipranol & Rispéridone & Vigabatrin \\
\hline Lisuride & Opium & Rivastigmine & Viloxazine \\
\hline Lithium & Oxcarbazépine & Rizatriptan & Vinblastine \\
\hline Loflazépate & Oxédrine & Ropinirole & Vincristine \\
\hline Loméfloxacine & Oxétorone & Scopolamine & Vindésine \\
\hline Loxapine (per os) & Oxitriptan & Sertindole & Voriconazole \\
\hline Maprotiline & Oxomémazine & Sertraline & Zolmitriptan \\
\hline Méclozine & Oxotomide & Sibutramine & Zonisamide \\
\hline Médazépam & Oxybutynine & Solifénacine & Zuclopebthixol (per os) \\
\hline $\begin{array}{l}\text { Médroxyprogestérone en } \\
\text { association }\end{array}$ & Oxycodone & Sterculia en association & \\
\hline Mémantine & Paclitaxel & Sulpride (per os) & \\
\hline Méphénésine & Paroxétine & Sultopride (per os) & \\
\hline Mépivacaïne & Péfloxacine & Sumatriptan & \\
\hline Méprobamate (per os) & Peginterféron & Tacrolimus & \\
\hline Mequitazine & Pémétrexed & Tégafur en association & \\
\hline Metformine et sulfamides & Penfluridol & Télithromycine & \\
\hline Méthadone & Pentazocine & Témozolomide & \\
\hline Méthocarbamol & Pentostatine & Tétrabénazine & \\
\hline Méthyldopa & Pergolide & Tétrazépam & \\
\hline Méthylphénidate & Périciazine & Tétryzoline & \\
\hline Métoclopramide & Péthidine & Thiopropérazine & \\
\hline Métopimazine & Phéniramine en association & Thioridazine & \\
\hline Miansérine & Phénobarbital & Tiagabine & \\
\hline Milnacipran & $\begin{array}{l}\text { Phényléphrine en } \\
\text { association }\end{array}$ & Tianeptine & \\
\hline Minocycline & Phénytoïne & Tiapride (per os) & \\
\hline Mirtazapine & Pilocarpine & Tobramycine & \\
\hline Mitotane & Piméthixène & Tofisopam & \\
\hline Mizolastine & Pimozide & Tolcapone & \\
\hline Moclobémide & Pipampérone & Toloxatone & \\
\hline Morphine & Pipotiazine (per os) & Toltérodine & \\
\hline
\end{tabular}


TABLEAU III : LISTE INDICATIVE DES SUBSTANCES DE NIVEAU 3SUSCEPTIBLESD'ALTERERLA CAPACITE DE LA CONDUITE AUTOMOBILE OU D’ENGINS PROFESSIONNELS (8).

\section{Liste alphabétique des substances avec pictogramme de niveau III}

\begin{tabular}{|c|c|c|c|}
\hline Alfentanil & Diazépam & Loprazolam & Ropivacaïne \\
\hline Alprazolam & Dropéridol & Lorazépam & Sévoflurane \\
\hline Amisulpride (forme parentérale) & Enflurane & Lormétazépam & Sodium oxybate \\
\hline Aripiprazole (forme parentérale) & Estazolam & $\begin{array}{c}\text { Loxapine (forme parentérale et } \\
\text { inhalée) }\end{array}$ & Stiripentol \\
\hline Atracurium & Ethanol & Médazépam & $\begin{array}{l}\text { Stiripentol (forme } \\
\text { parentérale) }\end{array}$ \\
\hline Atropine & Etomidate & Midazolam & Sufentanil \\
\hline Bromazépam & Fentanyl & Mivacurium & $\begin{array}{l}\text { Sulpiride (forme } \\
\text { parentérale) }\end{array}$ \\
\hline Brotizolam & Fluanisone (forme parentérale) & Nalorphine & $\begin{array}{l}\text { Sultopride (forme } \\
\text { parentérale) }\end{array}$ \\
\hline Bupivacaïne & Flumazénil & Niaprazine & Suxaméthonium \\
\hline Butobarbital & Flunitrazépam & Nitrazépam & Témazépam \\
\hline Chlordiazépoxyde & Flupentixol (forme parentérale) & Nordazépam & Thiopental \\
\hline Chloroprocaïne & $\begin{array}{l}\text { Fluphénazine (forme } \\
\text { parentérale) }\end{array}$ & Olanzapine (forme parentérale) & $\begin{array}{l}\text { Tiapride (forme } \\
\text { parentérale) }\end{array}$ \\
\hline Cisatracurium & Flurazépam & Oxazépam & Tofisopam \\
\hline Clobazam & Halopéridol (forme parentérale) & Pancuronium & Triazolam \\
\hline Clonazépam & Halothane & Perphénazine & Tropicamide \\
\hline Clorazépate & Homatropine & Phényléphrine & Vécuronium \\
\hline Clotiazépam & Isoflurane & Pipotiazine (forme parentérale) & Xénon \\
\hline $\begin{array}{c}\text { Cyamémazine (forme } \\
\text { parentérale) }\end{array}$ & Kétamine & Prazépam & Zolpidem \\
\hline Cyclopentolate & Lévobupivacaïne & Propofol & Zopiclone \\
\hline Desflurane & $\begin{array}{c}\text { Lévomépromazine (forme } \\
\text { parentérale) }\end{array}$ & Rémifentanil & $\begin{array}{c}\text { Zuclopenthixol } \\
\text { (forme parentérale) }\end{array}$ \\
\hline Dexmédétomidine & Loflazépate & Rocuronium & \\
\hline
\end{tabular}




\subsection{Autres substances psychoactives}

\subsubsection{Alcool}

Très souvent dans la société actuelle, l'impact de la consommation d'alcool éthylique reste sous-estimé. Pour certains, c'est un moyen de socialisation et en France, le vin apparait même comme un symbole de la tradition gastronomique reconnu dans le monde entier. Le chiffre d'affaire de la filière de l'alcool en France représentait 19,6 milliards d'euros en 2011 dont 15 milliards d'euros uniquement pour la viticulture, 2,5 milliards pour les spiritueux et 2,1 milliards pour la bière (15).

Selon l'ANPAA, l'alcool serait la substance psychoactive la plus consommée, avec seulement 7\% des individus de 18-75ans qui n'en auraient jamais consommé, 35\% des français qui en boivent régulièrement et $15 \%$ quotidiennement. Ceci représente $12,7 \mathrm{~L}$ d'alcool pur consommé par adulte par an en France. Les réactions à l'alcool sont dépendantes d'un individu à l'autre en fonction du poids, du sexe, de l'état de santé, de la quantité absorbée et du possible mélange avec d'autres substances (16). En effet, les hommes déclarent consommer en moyenne 2,8 verres d'alcool par jour ordinaire contre 1,7 verre pour les femmes. L'alcool le plus bu étant le vin, principalement suivi des spiritueux et de la bière. La consommation quotidienne est faible chez les 15-19 ans (1\%) contre 3\% chez les 20-25 ans. En revanche chez les plus de 25 ans, $15 \%$ déclarent boire de l'alcool quotidiennement. A contrario, la quantité consommée est plus importante chez les 15-25 ans (3,2 verres) que chez les plus de 25 ans (2,1 verres). En effet, les jeunes consomment moins fréquemment d'alcool mais cette consommation est généralement plus intense dans des contextes festifs. Chez les 15-25 ans les alcools « forts » et la bière sont plus consommés que le vin (16). Cela est en corrélation avec la mode du « binge drinking » que l'on pourrait traduire en français par « biture express ».Son principe est de boire de l'alcool ponctuellement, le plus souvent très rapidement et en grande quantité afin d'atteindre 
le plus vite possible un état d'ivresse, entrainant parfois des comas éthyliques voire dans certains cas des décès (17).

Plus globalement, selon la sécurité routière, l'alcool serait responsable d'un tiers des accidents mortels. Après avoir bu une personne a un risque multiplié par 8,5 d'être responsable d'un accident mortel. En 2016, parmi les accidents de la route impliquant un conducteur alcoolisé, 1009 personnes ont été tuées, 3500 personnes ont été hospitalisées, 2/3 des accidents ont lieu la nuit et $1 / 2$ les week-ends. On estime que 9 conducteurs sur 10 étaient des hommes et 25\% des 18-35 ans impliqués dans un accident étaient alcoolisés. L'alcoolémie délictuelle (supérieure ou égale à $0,8 \mathrm{~g} / \mathrm{L}$ de sang ou $0,40 \mathrm{mg} / \mathrm{L}$ d'air expiré) a augmenté de 2,2\%, représentant ainsi 22,3\% des délits (18).

\subsubsection{Stupéfiants}

On entend par stupéfiant toute « Substance toxique, médicamenteuse ou non, dont les propriétés sédatives, analgésiques, narcotiques et/ou euphorisantes provoquent une accoutumance et une pharmacodépendance (toxicomanie) » (19). Dans cette partie, il s'agira de traiter uniquement des « drogues » au sens strict du terme c'est-à-dire : « Substance psychotrope naturelle ou synthétique, généralement nuisible pour la santé, susceptible de provoquer une toxicomanie, et consommée en dehors d'une prescription médicale » (20) et plus précisément aux drogues illicites (opposées aux drogues dites« légales », réglementées, comme l'alcool, tabac et certains médicaments), dont la fabrication, la commercialisation et l'usage sont interdits par la loi, comme le cannabis, les opiacés (héroïne par exemple), la cocaïne, les amphétaminiques et les nouvelles drogues de synthèse dont le statut reste encore parfois mal défini. Ces substances ne sont pas systématiquement classées en fonction de leur dangerosité mais par rapport aux effets qu'elles entrainent : dépression respiratoire, stimulant, 
hallucinogène, stimulant-hallucinogène. En revanche, il existe dans chacune de ces familles de stupéfiants des substances plus ou moins dangereuses, associant des effets psychotropes ainsi qu'un risque de dépendance physique et/ou psychique plus ou moins marqué (21).

En France métropolitaine, sur une population de 11 à 75 ans le produit illicite le plus consommé est actuellement le cannabis. Il faut néanmoins distinguer le niveau de consommation, allant de l'expérimentation (au moins une fois dans sa vie), l'usage dans l'année (au moins une fois dans l'année en cours) à l'usage régulier (au moins 10 fois au cours du mois). En 2014, 17millions de français déclaraient avoir expérimenté au moins une fois dans la vie le cannabis, 2,2 millions la cocaïne, 1,7 millions l'ecstasy et 600000 l'héroïne. En ce qui concerne l'usage dans l'année, 4,6 millions pour le cannabis, 450000 pour la cocaïne et 400000 pour l'ecstasy. Enfin 1,4 million se déclaraient consommateurs réguliers de cannabis dont 700000 utilisateurs quotidiens. De plus on constate que le pourcentage d'expérimentateurs de substances illicites est le plus souvent compris dans la classe d'âge 26-34 ans (22).

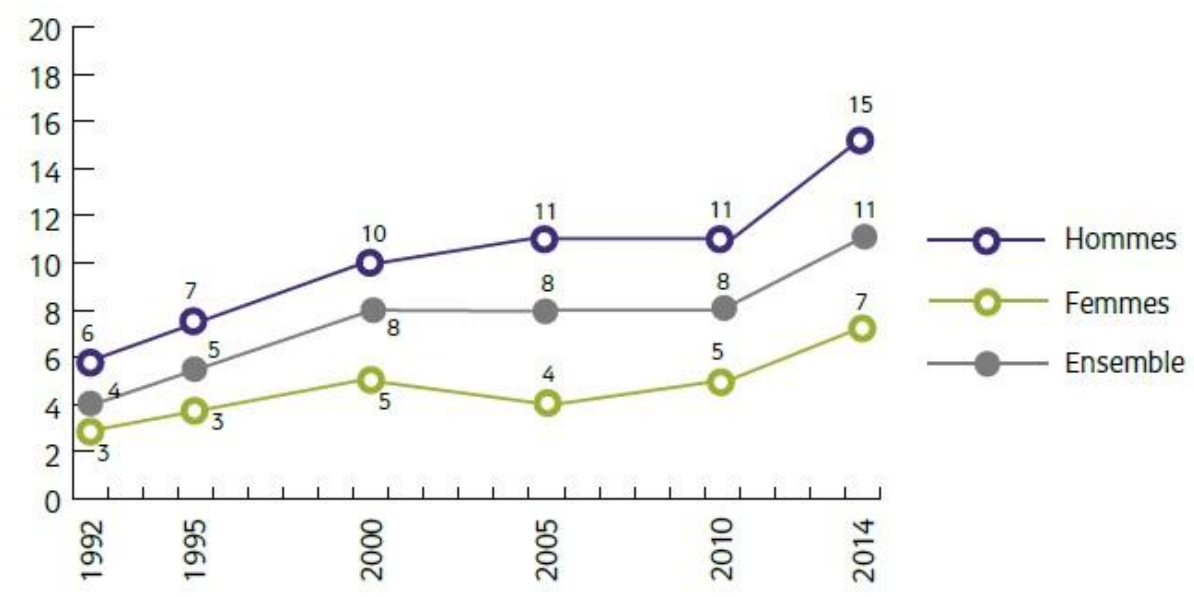
EN FRANCE, AU COURS DE L'ANNEE PARMI LES 18-64 ANS PAR SEXE (EN \%). (22) 
D'après la figure 8 issue d'un document de l'OFDT (22), on constate une progression quasi constante de la consommation de cannabis chez les 18-64 ans au cours des dernières années, quel que soit le sexe mais avec un pourcentage de consommateurs de sexe masculin deux fois plus élevé que celui des femmes.

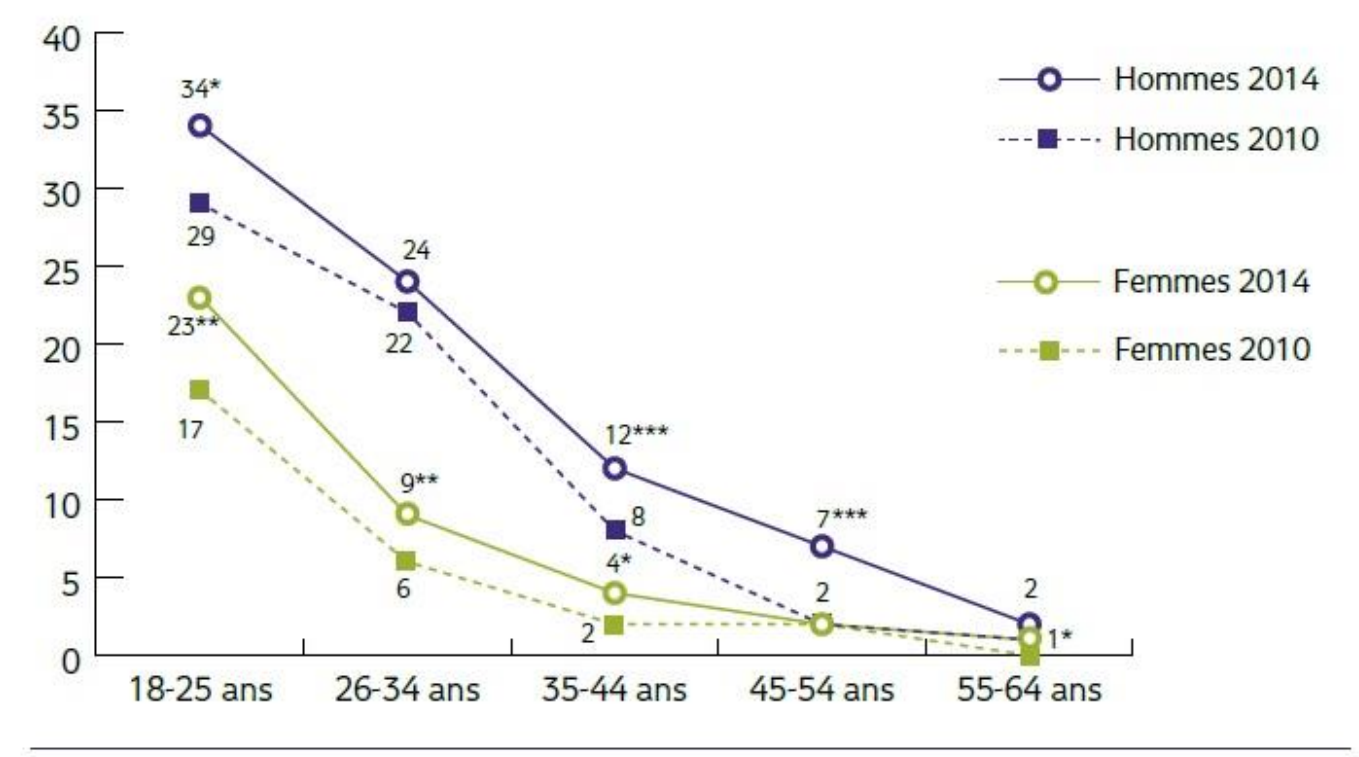

***, *** * : évolutions significatives aux seuils de 0,1 \%, $1 \%$ et $5 \%$ entre 2010 et 2014 , pour la classe d'áge concernée.

$(\%)(22)$.

Sur la figure 9 issue du même document, le pic de consommation de cannabis au cours de l'année se situe dans la classe d'âge 18-25 ans, $29 \%$ des hommes contre 17\% des femmes en 2010, pour décroitre régulièrement au fil des âges jusqu'à 1\% des hommes de 55-64 ans et moins de $1 \%$ pour les femmes de la même classe d'âge. En revanche l'usage de cannabis, toutes fréquences confondues, a augmenté entre 2010 et 2014 quel que soit le sexe, allant jusqu'à 34\% des hommes et 23\% des femmes de 18-25 ans en 2014 (étant toujours la tranche d'âge avec la consommation annuelle la plus élevée). Chez les femmes, on constate une chute du pourcentage dès la classe d'âge 26-34 ans passant ainsi de 23 à 9\% en 2014, pour décroitre ensuite plus 
progressivement. Chez les hommes, la décroissance est plus linéaire,24\% pour les 26-34 ans puis $12 \%$ pour les $35-44$ ans, $7 \%$ pour les $45-54$ ans et environ $2 \%$ pour les $55-64$ ans.

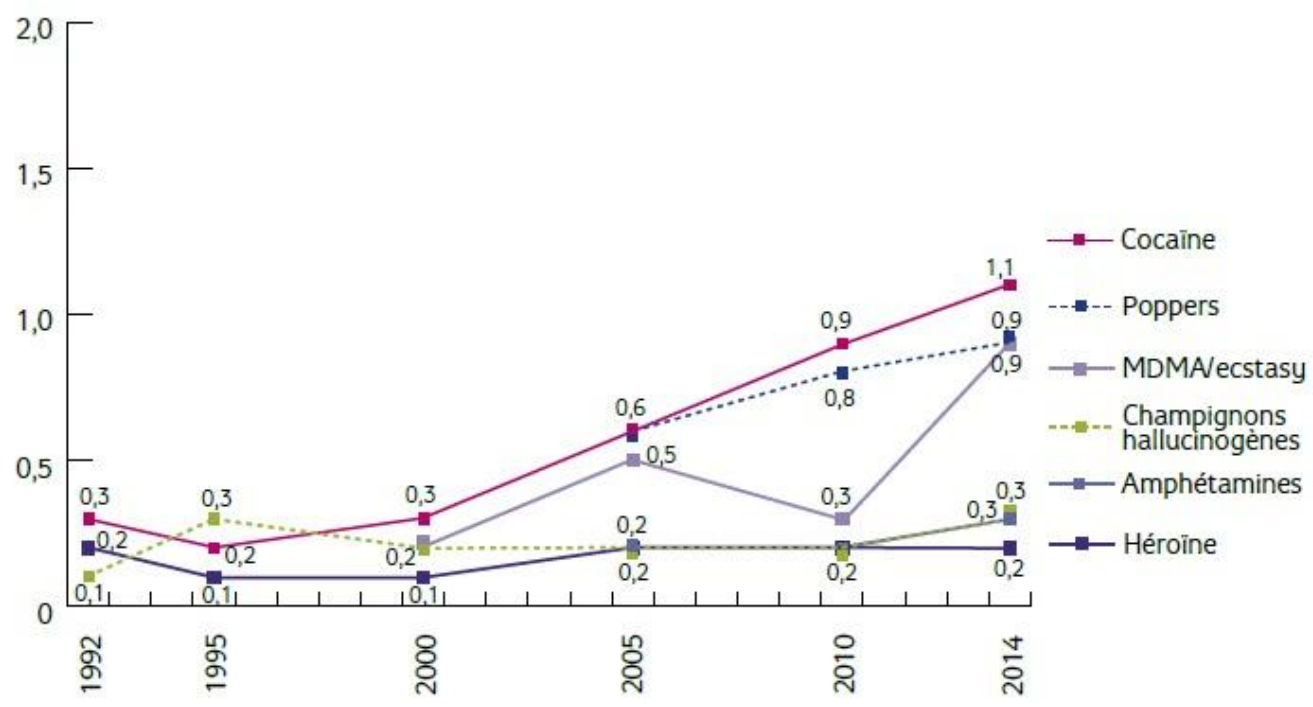

FIGURE 10 : EVOLUTION DE L'USAGE DANS L'ANNEE DES PRINCIPALES AUTRES DROGUES ILLICITES ENTRE 1992 ET 2014, PARMI LES 18-64 ANS (EN \%)(22).

Pour les autres drogues illicites, selon ce même document de l'OFDT (figure 10), on remarque que l'usage de cocaïne a été multiplié par un facteur 4 en 12 ans passant ainsi d'environ 0,3\% en 1992 à 1,1\% en 2014. Il en est de même avec les poppers, qui ne sont cependant plus considérés comme illégaux depuis le 3 juin 2013 (arrêté $\mathrm{n}^{\circ}$ 352484). La consommation de champignons hallucinogènes et d'héroïne est restée relativement stable entre 1992 et 2014 (environ 0,2\%). L'usage d'ecstasy (MDMA) a subi une augmentation entre 2000 et $2005(+0,3 \%)$ avant de rechuter jusqu'en $2010(-0,2 \%)$ et enfin ré-augmenter de manière significative (x3) et atteindre le même pourcentage que les poppers en 2014 c'est-à-dire 0,9\%. De manière plus globale, même si la consommation de ces autres substances illicites a augmenté au cours de ces dix dernières années, en dehors de la cocaïne (consommation en recrudescence 
chez les 17-50 ans en 2017, x10 en 20 ans) celle-ci reste globalement marginale par rapport à celle du cannabis (22).

\subsubsection{Usage détourné de médicaments psychoactifs}

L'effet de certains médicaments sur le système nerveux (soigner une douleur, un trouble anxieux, un trouble du sommeil, de l'humeur ou du comportement) a entrainé l'émergence d'usages détournés de certains médicaments, qui parfois pourrait être qualifié de récréatif, comme par exemple le «Purple Drank ». Ce cocktail utilisé par les jeunes adultes, à la popularité grandissante, contient une spécialité médicamenteuse codéinée et un antihistaminique, dilués dans un soda, provoquant ainsi un effet narcotique et psychodysleptique, associé à un ralentissement du rythme cardiaque. Des effets hallucinogènes peuvent être aussi recherchés avec le dextrométorphane avec dans certains cas des conséquences mettant en jeu le pronostic vital et nécessitant une hospitalisation (dépression respiratoire). Entre 2009 et 2013, 39 cas d'abus de dextrométorphane ont été recensés. En ce qui concerne l'association prométhazine / codéine, 35 cas d'abus recensés entre 2009 et 2015 dont environ 50\% entre janvier et août 2015. Une mise à jour des données fut faite en 2017 et indiquait que depuis janvier 2017, 5 cas d'intoxication au Purple Drank avaient été répertoriés dont 2 décès (23). C'est pourquoi depuis le 12 juillet 2017 certaines spécialités à base de codéine ou de dextrométorphane ont vu leur réglementation d'exonération modifiée, de manière à imposer une prescription médicale systématique (24).

La consommation de médicaments psychotropes est très répandue en France, comme développé dans le chapitre «1.1.1 Populations et consommation ». Il a été possible de constater un détournement de l'usage de ces médicaments en partie chez les adultes, mais aussi par « une 
culture jeune qui normalise les usages récréatifs de ces substances ». Les facteurs d'entrée en consommation sont principalement la prescription médicale, et l'âge (correspondant généralement à la période d'émancipation liée à l'envie de nouvelles expérimentations). Par ailleurs, l'automédication, la redéfinition par le patient des schémas posologiques initialement prescrits peuvent conduire à un mésusage des médicaments, une utilisation à des fins récréatives et exposent à un risque d'addiction (25).

C'est pour toutes ces raisons que l'ANSM, en plus du suivi des effets indésirables (pharmacovigilance), a mis en place une surveillance des abus, des dépendances et des usages détournés via l'addictovigilance avec un réseau régional : les Centre d'Evaluation et d'Information sur la Pharmacodépendance (CEIP) permettant ainsi la surveillance de l'utilisation de produits psychoactifs, ainsi que le potentiel risque d'abus/dépendance. Sur le site de l'ANSM (https://www.ansm.sante.fr/Activites/Surveillance-des-stupefiants-et-despsychotropes/Medicaments-a-risque-d-usage-detourne-ou-de-dependance/(offset)/0) il est possible de trouver un récapitulatif (figure 11) sur la classe des médicaments et les motifs de surveillance (26).

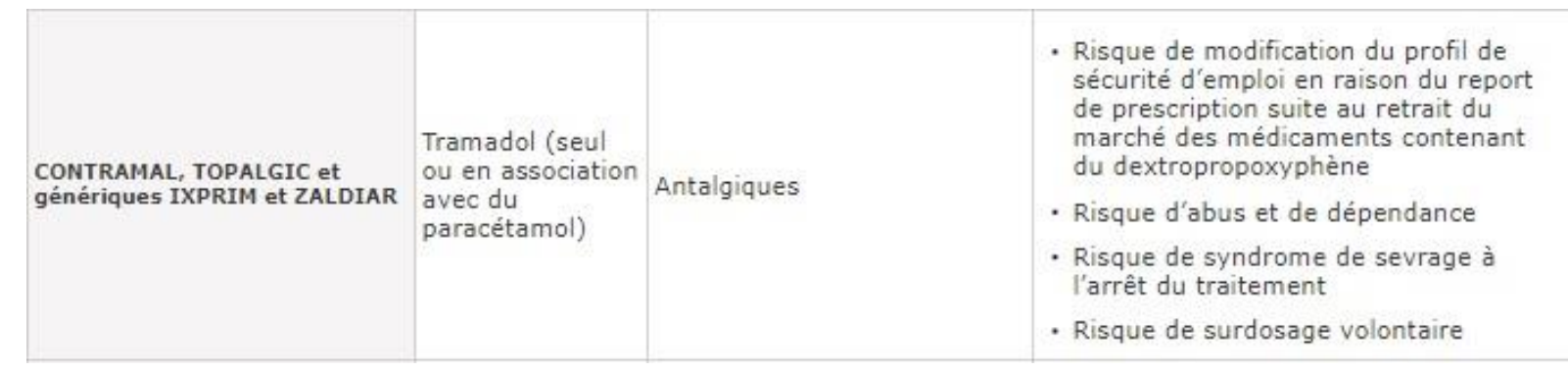




\subsection{Accidents de la voie publique liés à la consommation de produits}

psychoactifs en France

En France, la première cause d'accidents mortels sur la route reste la vitesse excessive ou inadaptée, mais en deuxième place on retrouve l'usage de substance(s) psychoactive(s) telles que l'alcool, les produits stupéfiants ou les médicaments. Les causes d'accidents mortels varient en fonction des classes d'âge, même si la vitesse semble être dominante dans la majorité des cas. Chez les 18-24ans et les 25-34 ans, la consommation d'alcool est retrouvée dans 23\% des cas chez les 18-24 ans et 27\% chez les 25-34 ans. L'usage de produits stupéfiants est retrouvé dans environ $13 \%$ des accidents routiers chez les 18-24 ans et 15\% pour les 25-34 ans. Au-delà de 35 ans, en plus de ces facteurs de risque, on retrouve également des troubles du comportement tels que des refus de priorités, des inattentions et des malaises (27). Ces facteurs de risques en fonction des classes d'âges sont présentés dans la Figure 12, pour la période 20142017 en France. Le rôle des médicaments dans la survenue d'accidents de la route reste cependant sous-estimé. 


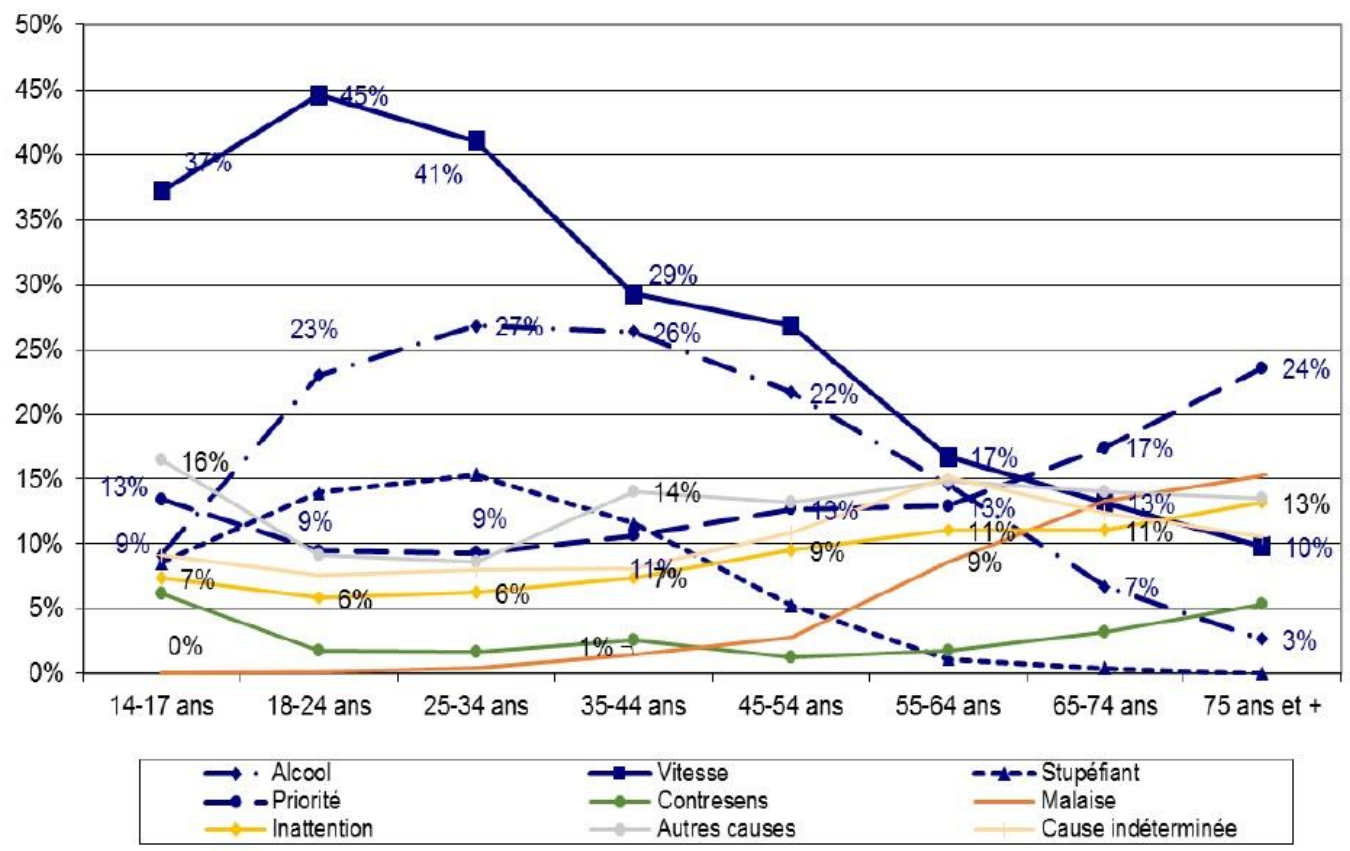

FIGURE 12 : FACTEURS COMPORTEMENTAUX CHEZ LES AUTEURS PRESUMES D'ACCIDENTS MORTELS DE SEPTEMBRE 2014 A DECEMBRE 2017 (26).

Sur le réseau routier bidirectionnel hors agglomération en 2017, la vitesse, l’alcool et les stupéfiants apparaissaient comme étant les principales causes d'accidents mortels chez les 18-24 et les 25-34 ans. On retrouve un pic proche de 30\% pour l'alcool chez les $20-45$ ans (Figure 13).

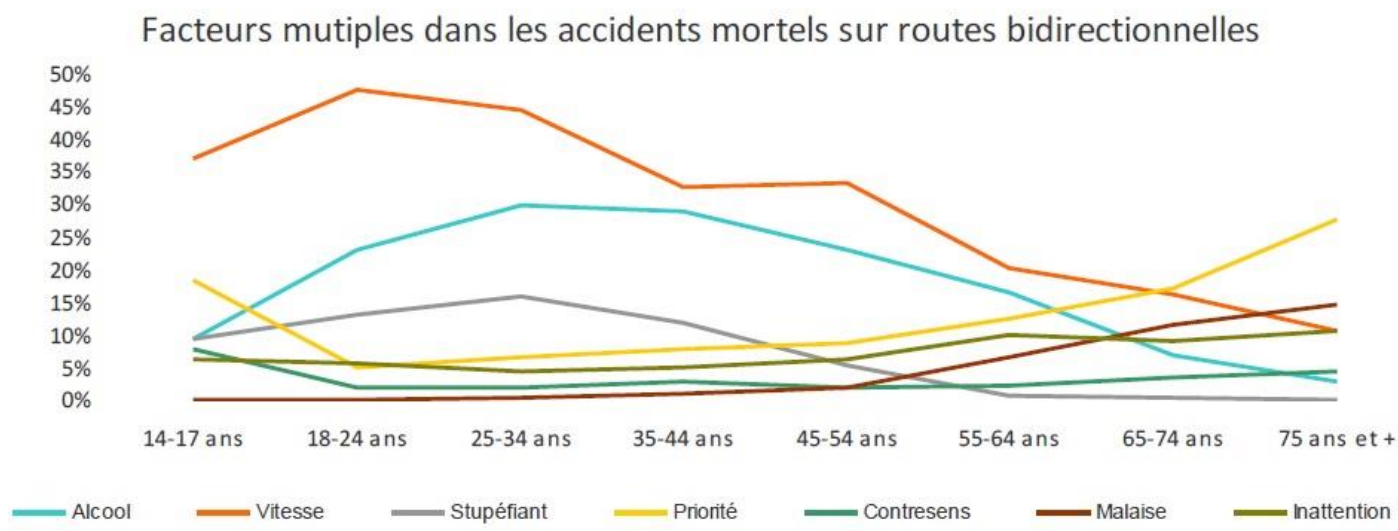

FIGURE 13 : FACTEURS COMPORTEMENTAUX CHEZ LES AUTEURS PRESUMES D'ACCIDENTS MORTELS SUR ROUTES BIDIRECTIONNELLES HORS AGGLOMERATIONS EN 2017 (26). 
Sur les routes des agglomérations, la consommation d'alcool est également présente, avec un pourcentage sensiblement identique aux données précédentes, mais l'implication des stupéfiants est moindre que les refus de priorité, avec toujours la même proportion de $15 \%$ environ et quasiment nulle après 55 ans (Figure 14).

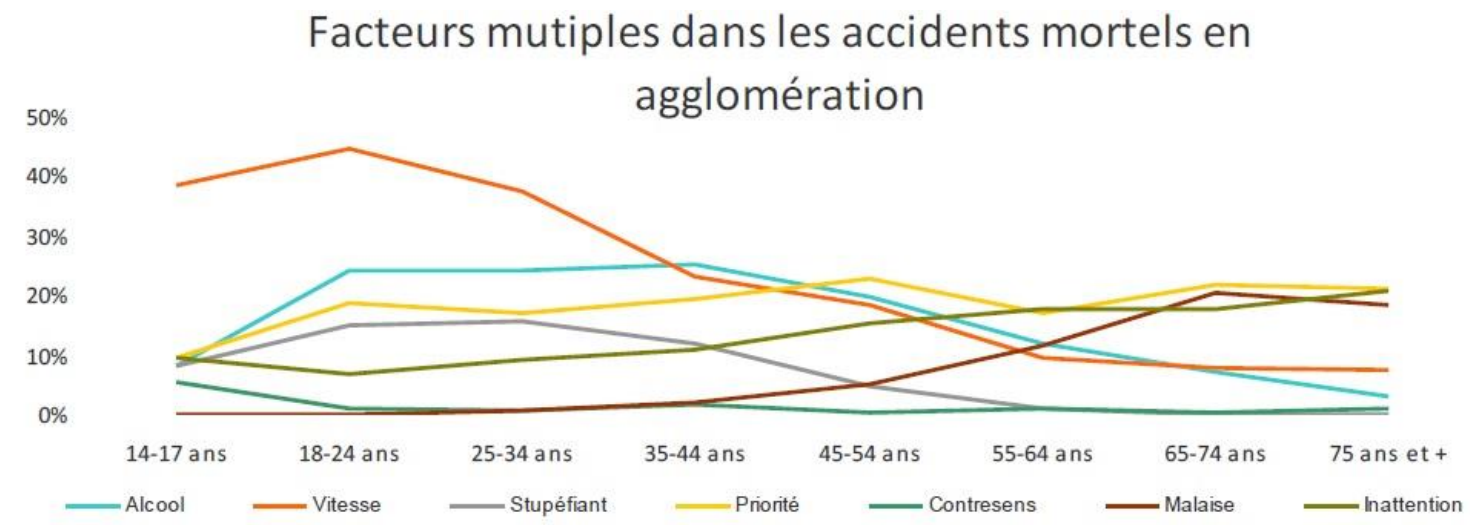

FIGURE 14 : FACTEURS COMPORTEMENTAUX CHEZ LES AUTEURS PRESUMES D'ACCIDENTS MORTELS EN AGGLOMERATIONS (26).

En ce qui concerne la consommation de stupéfiants, on estime qu'elle était à l'origine de 752 tués en 2016 sur les routes. La classe d'âge la plus concernée est celle des 25-34 ans (22\%) et dans 1 accident sur 5 le conducteur en avait consommé. Dans 93\% des cas ce sont des hommes, qui sont contrôlés positifs dans les accidents mortels (28).

Il n'existe que peu de données concernant la prévalence et le rôle des médicaments dans les accidents de la voie publique. Une étude publiée en 2003 a été menée en France sur 900 personnes impliquées dans un accident de la route et 900 autres personnes soumise à un simple contrôle routier, entre juin 2000 et septembre 2001. Cette étude a montré une prévalence plus importante des consommations d'alcool, de stupéfiants (cannabinoïdes principalement et autres) ou d'autres molécules psychoactives (dont les médicaments) chez les victimes 
d'accidents de la route par rapport au groupe contrôle. Ceci suggère un rôle important de l'ensemble de ces substances dans la survenue d'accidents de la route. Parmi les médicaments concernés on retrouve de loin les benzodiazépines (benzodiazépines hypnotiques exclues) : prévalence de $14 \%$ dans le groupe des impliqués dans un accident et 12,6\% dans le groupe contrôle, suivis par les antidépresseurs avec une prévalence de l'ordre de 1 à $2 \%$ (29).

Au final, chaque substance psychoactive présente un risque potentiel pour la conduite automobile avec des degrés plus ou moins importants en fonction des doses consommées et des classes thérapeutiques. Ce risque reste cependant mal évalué et sous-estimé, il est d'ailleurs régulièrement méconnu des patients du fait d'une information insuffisante.

D'autre part, l'association de plusieurs substances psychoactives (médicaments, stupéfiants et/ou alcool) potentialisent davantage le risque pour les conducteurs d'être impliqués dans un accident. 


\section{Travaux Personnels : étude rétrospective d'une série de cas au CHU de Rouen.}

\subsection{Contexte}

En France, les analyses toxicologiques réalisées dans un contexte médico-légal, tel qu'un accident de la route ou encore une recherche des causes de la mort, sont généralement réalisées par des biologistes médicaux déclarés « experts » par leur tribunal ou cours d'appel de rattachement. Ces analyses sont effectuées sur réquisitions judiciaires rédigées par des officiers de police judiciaire (gendarmes ou agents de police habilités et qui agissent sur ordre du procureur de la république), ainsi que sur ordonnance de commission d'expert à la demande directe d'un magistrat (le plus souvent juge d'instruction ou procureur). Cette prescription d'analyses toxicologique est à la discrétion de l'enquêteur, en fonction du contexte de chaque affaire. Les analyses toxicologiques peuvent comprendre une recherche et dosage de l'alcool (éthanol) dans le sang, associée ou non à une recherche de produits stupéfiants et d'autres toxiques tels que des médicaments par exemple. Dans le cadre des accidents de la voie publique, ces analyses ou expertises se limitent le plus souvent à la recherche isolée de l'éthanol dans le sang et/ou des principaux produits stupéfiants dans le sang ou la salive.

D'après l'arrêté du 29 septembre 2017(30) et une note de novembre 2017 de la Garde des Sceaux (31), l'expertise toxicologique dite «de référence» comporte généralement :

- La recherche d'une exposition aux alcools. Identification et dosage de l'éthanol, méthanol et autres alcools par CPG, ionisation de flamme et autres techniques chromatographiques.

- Le Dosage de la carboxyhémoglobine dans le sang.

- La recherche et le dosage des cyanures selon contexte du décès. 
- La recherche large (criblage toxicologique ou « screening ») par technique chromatographique : CPG couplée à la spectrométrie de masse, chromatographie liquide couplée à une barrette de diodes, et/ou à la spectrométrie de masse en tandem, et/ou à la spectrométrie de masse haute résolution.

- La recherche et le dosage des produits stupéfiants (cannabinoïdes, amphétaminiques, cocaïne, opiacés, leurs principaux métabolites et substances apparentées), des produits de substitution aux toxicomanies et des nouveaux produits de synthèses (NPS) par chromatographie avec détection par spectrométrie de masse.

- L’identification et dosage des médicaments psychoactifs (hypnotiques, anxiolytiques, neuroleptiques et antidépresseurs) ainsi que leurs principaux métabolites par chromatographie avec détection par spectrométrie de masse.

- L'identification et dosage des médicaments non psychoactifs ainsi que leurs métabolites (bêta-bloquants, antiarythmiques, anesthésiques, anticoagulants, antidiabétiques, antalgiques, antiparkinsoniens) et de xénobiotiques divers par chromatographie avec détection par spectrométrie de masse.

- La recherche et dosage de lithium selon le contexte du décès.

Cependant, dans la pratique courante, en cas de délits routiers, à la demande des services de police ou de gendarmerie il est fréquent de ne retrouver que la recherche et le dosage d'éthanol ainsi que la recherche et le dosage des stupéfiants. Les médicaments restent actuellement rarement recherchés dans les contextes d'accidents de la voie publique et délits routiers. Dans ces contextes, cela dépend de la gravité des faits et s'il y a des dommages corporels. Dans le cas d'un accident sans gravité, éthanol +/- stupéfiants seront principalement 
recherchés, et dans certains cas particuliers il s'agira d'une expertise toxicologique de référence telles que détaillée ci-dessus, incluant notamment les médicaments psychoactifs.

\section{$2.2 \quad$ Objectifs}

Les objectifs de ce travail étaient d'évaluer l'incidence des médicaments ou substances médicamenteuses associés à un risque reconnu pour la conduite automobile dans un groupe d'individus ayant fait l'objet d'un contrôle routier dans un contexte d'infraction ou d'AVP (accident de la voie publique), au moyen d'un screening toxicologique employé au laboratoire de Pharmacologie-Toxicologie et de Pharmacogénétique du CHU de Rouen. La méthodologie employée au cours de ce travail comprend la recherche ciblée de plus de 230 molécules par chromatographie en phase liquide couplée à la spectrométrie de masse en tandem (HPLC- ou LC-MS/MS). Au cours de ce travail, nous avons retenu dans cette méthode la recherche d'une centaine de substances, pour lesquelles un pictogramme existe, incluant également les principaux produits stupéfiants.

Un objectif secondaire de ce travail était d'évaluer quelles classes de médicaments «à risque »sont le plus fréquemment retrouvées, alors que leur recherche n'était pas demandée sur la réquisition judiciaire initiale. Enfin, nous tentons de déterminer un score pour évaluer l'importance de ce risque en fonction des associations entre un (ou plusieurs) médicament(s) et les « toxiques » habituellement recherchés par défaut (stupéfiants et/ou alcool). 


\subsection{Matériels et méthodes}

\subsubsection{Populations / échantillons étudiés}

Au cours de cette étude, les échantillons de sang retenus pour l'analyse étaient ceux provenant d'individus figurants parmi la banque d'échantillons disponibles dans le Laboratoire de Pharmacologie - Toxicologie et de Pharmacogénétique du CHU de Rouen. En effet, ce laboratoire propose une activité de toxicologie médico-légale et les échantillons analysés concernaient ceux de l'année 2016 (du 01 janvier 2016 au 31 décembre 2016), pour lesquels une recherche et/ou dosage d'éthanol et/ou de stupéfiants était demandé sur réquisition judiciaire. Aucun autre filtre, tel que le sexe ou l'âge, n'a été appliqué. Afin de s'assurer que l'analyse portait uniquement sur des conducteurs automobile, nous avons vérifié que chaque échantillon sélectionné pour l'étude provenait d'une personne identifiée comme conducteur(trice) au moment du contrôle et/ou de l'accident de la voie publique. Dans un souci de logistique et de coût, le nombre d'individus sélectionnés a été limité à 100. Après anonymisation des échantillons, l'analyse a été faite à partir d'un échantillon de sang.

\subsubsection{Méthodologie Analytique}

\subsubsection{Généralités}

Lors de ce travail, le dépistage qualitatif et quantitatif des différentes molécules a été effectué au moyen d'un «screening toxicologique », correspondant par définition à une recherche large et simultanée de plus de 230 substances (médicaments, métabolites, toxiques et stupéfiants). Pour ce screening la technique de choix est l'HPLC (ou CLHP-MS/MS pour chromatographie liquide à haute performance couplée à la spectrométrie de masse en tandem) 
et la recherche et le dosage de l'éthanol est réalisée par chromatographie en phase gazeuse couplée à un détecteur à ionisation de flamme (GC- ou CPG-FID).

La chromatographie est une technique permettant de séparer plusieurs composés dans un même mélange afin de les identifier, et de les quantifier. En chromatographie « liquide » ou « HPLC », la phase mobile, c'est-à-dire la phase traversant le système chromatographique est liquide et sous haute pression (soit « haute performance » ou« high performance » en anglais) permettant ainsi d'augmenter le débit et l'efficacité de la séparation des composés. Les composés à analyser (analytes) sont en solution dans un solvant ou un milieu biologique, comme le sang, qui est lui-même injecté dans la phase mobile et traverse donc le système chromatographique. Ce système chromatographique comprend une colonne séparative de composition variable, choisie en fonction de la nature des analytes à séparer. Les analytes se répartissent sur cette colonne ou phase « stationnaire » en fonction de leur affinité physicochimique pour les composants de celle-ci (notamment polarité, charge ionique, structure chimique, hydrophobicité ou lipophilie et taille) entre la phase mobile et la phase stationnaire du système chromatographique. Cette interaction entre les analytes et ces deux phases du système permet leur séparation en fonction de leurs caractéristiques physico chimiques (Figure $15)$.

Le système chromatographique est systématiquement couplé à un détecteur, également adapté aux caractéristiques des analytes recherchés (dans le cas de cette étude il s'agit d'un spectromètre de masse en tandem), qui permet de caractériser chacun des solutés par un signal ou « pic chromatographique » qui lui est propre dans des conditions données (temps de rétention, masse et fragmentation de cette masse caractéristiques). La phase stationnaire de la colonne chromatographique est constituée de nombreuses particules de silice, de forme sphérique et sur lesquelles sont généralement greffés des groupements carbonés destinés à 
retenir les analytes de manière plus ou moins marquée en fonction de leur affinité physicochimique avec ceux-ci. Plus leur granulométrie diminue, plus les particules de la phase stationnaire sont nombreuses et donc plus il y a d'interactions, associées à une augmentation de la «résistance» à l'écoulement de la phase mobile et donc de pression (32).

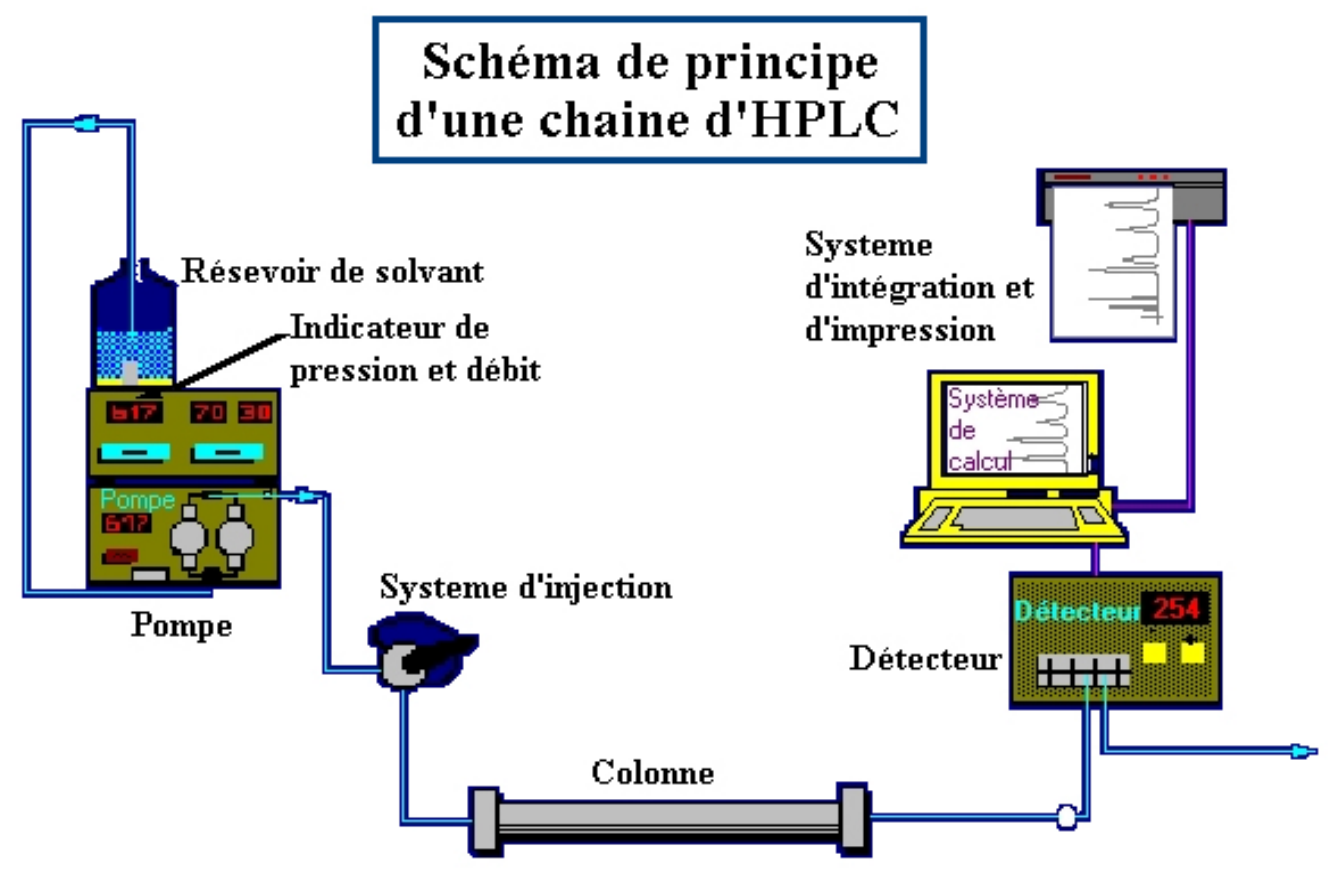

FIGURE 15 : REPRESENTATION SCHEMATIQUE D'UN SYSTEME HPLC (32).

La pompe permet de faire circuler le solvant (ou phase mobile) en continu dans le système chromatographique à débit constant et haute pression.

Le système d'injection permet d'introduire l'échantillon à analyser dans la phase mobile et en direction de la colonne séparative. Les systèmes les plus couramment utilisés sont équipés d'une vanne avec une boucle d'échantillonnage dont le volume est fixe, afin de maintenir une pression équilibrée dans l'ensemble du système et d'assurer la répétabilité du volume injecté. Dans un premier temps l'échantillon est injecté dans cette boucle via un injecteur réglé sur un volume fixe. La vanne permet ensuite le passage de l'échantillon vers la phase mobile. 
La colonne chromatographique utilisée est caractérisée par une longueur, un diamètre interne, une taille et une composition des particules la composant. Elle est choisie par l'utilisateur en fonction des objectifs analytiques (tels que nature du ou des analytes à rechercher, temps d'analyse, contraintes de pression liées au système, qualité des pics chromatographiques ou encore la sensibilité analytique ciblée).

Le détecteur permet de transcrire un signal dont l'intensité est idéalement directement proportionnelle à la concentration du ou des analytes à la sortie du système chromatographique (Figure 15). Au cours de ce travail, l'HPLC a été couplée à un détecteur de type spectromètre de masse en tandem : les molécules contenues dans l'échantillon sont désolvatées à haute température puis ionisées sous pression atmosphérique à l'aide d'un champ électrique (méthode dite de l'électrospray permettant de charger soit positivement soit négativement l'analyte). La molécule ionisée est ensuite orientée vers un système triple quadripôle. Un quadripôle est constitué de quatre barreaux électromagnétiques dont la polarité (positif/négatif) s'alterne en continu, afin de permettre la séparation et la migration des analytes chargés vers une cellule de collision assurant leur détection, en fonction de leur rapport masse sur charge $(\mathrm{m} / \mathrm{z})$. Les analytes ainsi filtrés sont ensuite soumis à une fragmentation dans une cellule de collision qui permet de générer des fragments caractéristiques et un spectre de masse (spectrométrie de masse en tandem) analysé par un dernier quadripôle. La dernière étape consiste à l'intégration de ce signal sous la forme de pics chromatographique dont la surface et/ou l'intensité sont proportionnelles à la concentration initiale de l'analyte dans l'échantillon biologique. 


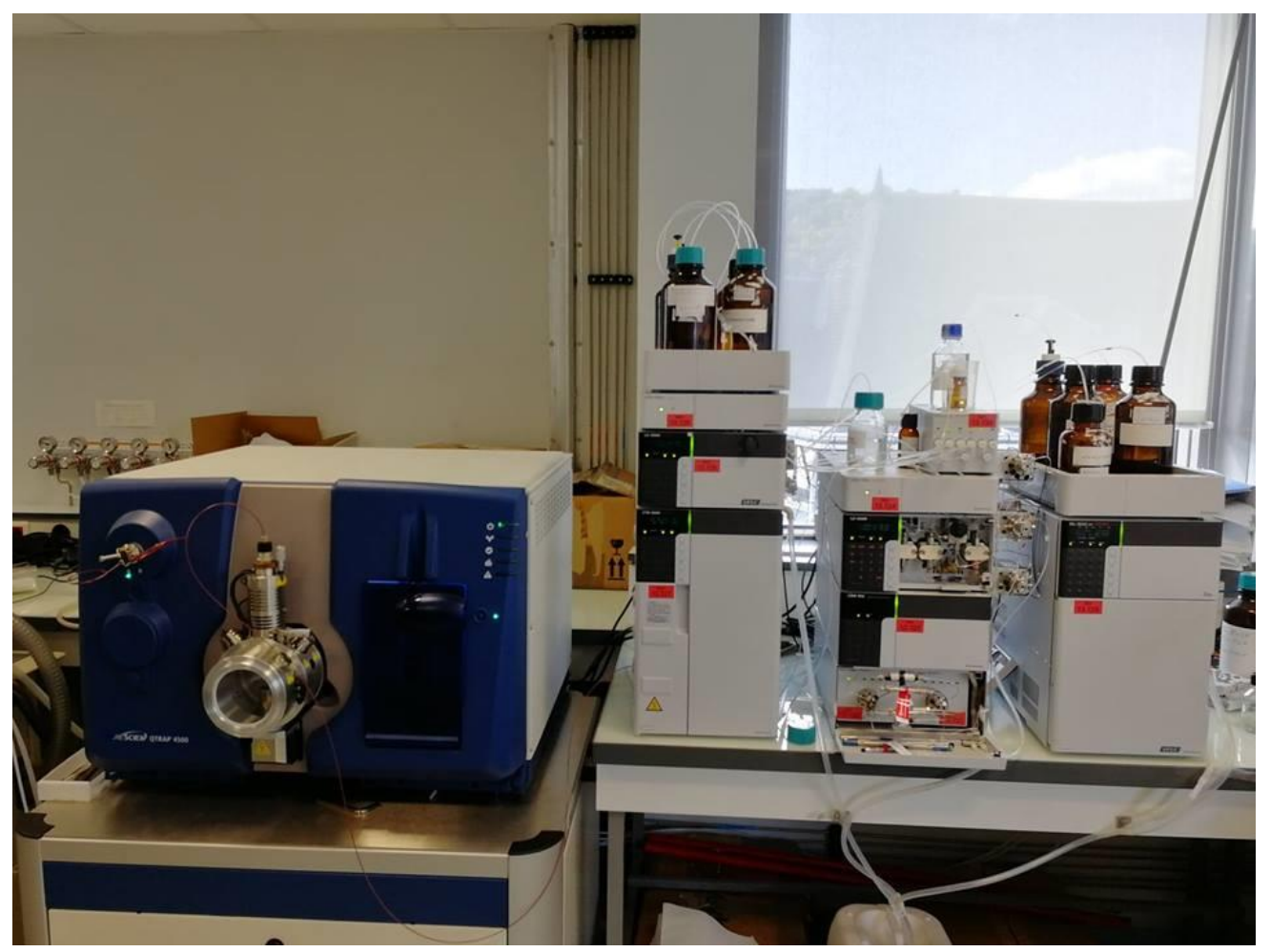

FIgURE 16 : PHOTOGRAPHIE DU SYSTEME HPLC-MS/MS UTILISE AU COURS DE CE TRAVAIL.

La figure 16 est une photographie du système chromatographique (à droite) couplé au spectromètre de masse en tandem (à gauche) du laboratoire de pharmacologie-toxicologie du CHU de Rouen et utilisé pour ce travail. 


\subsubsection{Préparation de l'analyte}

Dans un premier temps des étalons (calibrateurs) sont préparés, ce sont des solutions contenant les substances de référence que l'on souhaite analyser à une concentration connue, le plus souvent préparées à partir de solutions à $1 \mathrm{mg} / \mathrm{mL}$ dans le cas présent. Par exemple, un calibrateur «antidépresseurs » contient plusieurs molécules de référence : clomipramine, amitriptyline, citalopram, duloxétine, etc. Pour cette technique, le laboratoire dispose de plusieurs calibrateurs en fonction des classes thérapeutiques, telles que benzodiazépines, bêtabloquants et autres cardiotropes, antibiotiques, neuroleptiques, stupéfiants et des calibrateurs mixtes contenant différentes molécules ne pouvant être mises dans les classes précédentes comme certains antidiabétiques, anti-infectieux, médicaments à visée cardiovasculaire, etc.

Les analyses ont été effectuées à partir d'un échantillon de sang mais il est possible de les effectuer également à partir de différentes matrices biologiques (urines, sérum, plasma, ...).

Pour la préparation des échantillons, une prise d'essai de $100 \mu \mathrm{L}$ de sang est déposée dans un cône de type eppendorf ${ }^{\circledR}$. Une solution de sulfate de zinc contenant un étalon interne est ajoutée, notamment afin d'hémolyser l'échantillon et de rompre les liaisons des analytes aux protéines, lipides et autres macromolécules. Le milieu réactionnel est agité au vortex puis centrifugé. Le surnageant est directement injecté dans l'analyseur.

Ce mode opératoire a été répété pour chaque série analytique qui comprend: un «blanc », c'est-à-dire un échantillon de sang sans analyte, le ou les échantillons à analyser « purs », ainsi que dilués au 1/10 ème et des calibrateurs à $10 \mathrm{ng} / \mathrm{mL}$ et $100 \mathrm{ng} / \mathrm{mL}$. 
TABLEAU IV : LiSTE DES MOLECULES D'INTERETS RECHERCHEES PAR SCREENING AVEC LEURS TEMPS DE RETENTION (TR) ET UN NIVEAU DE VIGILANCE NON DEFINI.

\begin{tabular}{ccc}
\hline Analyte & $\begin{array}{c}\text { TR } \\
\text { théo }\end{array}$ & Vigilance conduite \\
\hline Aciclovir & 1,0 & Non défini \\
\hline $\begin{array}{c}\text { Amoxicilline }+ \text { Ac } \\
\text { Clav }\end{array}$ & 1 & Non défini \\
\hline Atenolol & 1,8 & Non défini \\
\hline Bisoprolol & 3,9 & Non défini \\
\hline Carvedilol & 4,4 & Non défini \\
\hline Clarithromycine & 0 & Non défini \\
\hline Diltiazem & 4,4 & Non défini \\
\hline Fluconazole & 4 & Non défini \\
\hline Griseofulvine & 0 & Non défini \\
\hline
\end{tabular}

\begin{tabular}{ccc}
\hline Analyte & $\begin{array}{c}\text { TR } \\
\text { théo }\end{array}$ & Vigilance conduite \\
\hline Hydroxy-itraconazole & 6 & Non défini \\
\hline Imipenem & 1 & Non défini \\
\hline Indapamide & 4 & Non défini \\
\hline Itraconazole & 6 & Non défini \\
\hline Lercanidipine & 5 & Non défini \\
\hline Nebivolol & 5 & Non défini \\
\hline Quinidine & 3 & Non défini \\
\hline Thebacon & 0 & Non défini \\
\hline Thebaine & 0 & Non défini \\
\hline
\end{tabular}

TABLEAU V : LISTE DES MOLECULES D'INTERETS RECHERCHEES PAR SCREENING AVEC LEURS TEMPS DE RETENTION (TR) ET UN NIVEAU DE VIGILANCE 1.

\begin{tabular}{ccc}
\hline Analyte & $\begin{array}{c}\text { TR } \\
\text { théo }\end{array}$ & $\begin{array}{c}\text { Vigilance } \\
\text { conduite }\end{array}$ \\
\hline Amlodipine & 4,6 & 1 \\
\hline Buspirone & 3,9 & 1 \\
\hline Ceftazidime & 1 & 1 \\
\hline Cetirizine & 4,8 & 1 \\
\hline Chloroquine & 2,1 & 1 \\
\hline Cibenzoline & 0 & 1 \\
\hline Codeine & 2 & 1 \\
\hline Dextromethorphane & 0 & 1 \\
\hline Diclofenac & 0 & 1 \\
\hline Disopyramide & 3,6 & 1 \\
\hline
\end{tabular}

\begin{tabular}{ccc}
\hline Analyte & $\begin{array}{c}\text { TR } \\
\text { théo }\end{array}$ & Vigilance conduite \\
\hline Ertapenem & 1 & 1 \\
\hline Ethylmorphine & 3 & 1 \\
\hline Felodipine & 6 & 1 \\
\hline Flecainide & 4 & 1 \\
\hline Meropenem & 2 & 1 \\
\hline Norcodeine & 2 & 1 \\
\hline Papaverine & 4 & 1 \\
\hline Pholcodine & 2 & 1 \\
\hline Pseudoephedrine & 0 & $1 / 2 / 3$ \\
\hline Urapidil & 3 & 1 \\
\hline
\end{tabular}


TABLEAU VI : LiSTE DES MOLECUlES D'INTERETS RECHERCHEES PAR SCREENING AVEC LEURS TEMPS DE RETENTION (TR) ET UN NIVEAU DE VIGILANCE 2.

\begin{tabular}{|c|c|c|}
\hline Analyte & $\begin{array}{l}\text { TR } \\
\text { théo }\end{array}$ & $\begin{array}{l}\text { Vigilance } \\
\text { conduite }\end{array}$ \\
\hline Alimemazine & 4,5 & 2 \\
\hline Amisulpride & 2,8 & 2 \\
\hline Amitriptyline & 4,5 & 2 \\
\hline Amoxapine & 4,4 & 2 \\
\hline Aripiprazole & 4,6 & 2 \\
\hline Baclofene & 1 & 2 \\
\hline Buprenorphine & 4,2 & 2 \\
\hline Buprenorphine-Glu & 3,7 & 2 \\
\hline Carbamazepine & 4,6 & 2 \\
\hline $\begin{array}{c}\text { Carbamazepine-10-11- } \\
\text { epoxide }\end{array}$ & 0 & 2 \\
\hline Chlorediazepoxide & 4,1 & 2 \\
\hline Chlorpromazine & 0 & 2 \\
\hline Ciprofloxacine & 3 & 2 \\
\hline Citalopram & 4,1 & 2 \\
\hline Clomipramine & 4,8 & 2 \\
\hline Clonidine & 2,2 & 2 \\
\hline Clozapine & 3,9 & 2 \\
\hline Cyamemazine & 4,4 & 2 \\
\hline Desmethyl-clozapine & 3,8 & 2 \\
\hline Dihydrocodeine & 2 & 2 \\
\hline Diphenhydramine & 4,1 & 2 \\
\hline Dosulepine & 4,3 & 2 \\
\hline Doxepine & 4,1 & 2 \\
\hline Duloxetine & 4,5 & 2 \\
\hline EDDP & 4 & 2 \\
\hline
\end{tabular}

\begin{tabular}{|c|c|c|}
\hline Analyte & $\begin{array}{c}\text { TR } \\
\text { théo }\end{array}$ & Vigilance conduite \\
\hline Fentanyl & 4 & 2 \\
\hline Fluoxetine & 5 & 2 \\
\hline Flupentixol & 0 & 2 \\
\hline Fluvoxamine & 5 & 2 \\
\hline Gabapentine & 1 & 2 \\
\hline Ganciclovir & 1 & 2 \\
\hline Glibenclamide & 6 & 2 \\
\hline Gliclazide & 5 & 2 \\
\hline Glimepiride & 6 & 2 \\
\hline Glipizide & 5 & 2 \\
\hline Haloperidol & 4 & $2 / 3$ \\
\hline Hydromorphone & 2 & 2 \\
\hline Hydroxyzine & 5 & 2 \\
\hline Imipramine & 4 & 2 \\
\hline Lamotrigine & 3 & 2 \\
\hline Levetiracetam & 2 & 2 \\
\hline Levofloxacine & 3 & 2 \\
\hline Levomepromazine & 5 & 2 \\
\hline Lidocaine & 3 & 2 \\
\hline Loxapine & 4 & 2 \\
\hline Maprotiline & 5 & 2 \\
\hline Methadone & 5 & 2 \\
\hline Metopimazine & 0 & 2 \\
\hline Metopimazine acide & 0 & 2 \\
\hline Mianserine & 4 & 2 \\
\hline
\end{tabular}




\begin{tabular}{|c|c|c|}
\hline Milnacipran & 4 & 2 \\
\hline Mirtazapine & 3 & 2 \\
\hline Moclobemide & 3 & 2 \\
\hline Morphine & 2 & 2 \\
\hline Morphine-3\&6-beta-glu & 2 & 2 \\
\hline $\mathrm{N}$-desmethyltramadol & 3 & 2 \\
\hline Nefopam & 4 & 2 \\
\hline Norbuprenorphine & 4 & 2 \\
\hline Norbuprenorphine-Glu & 3 & 2 \\
\hline Norclomipramine & 5 & 2 \\
\hline Norfentanyl & 3 & 2 \\
\hline Norfluoxetine & 5 & 2 \\
\hline Norsertraline & 5 & 2 \\
\hline Nortriptyline & 5 & 2 \\
\hline Norvenlafaxine & 4 & 2 \\
\hline Noscapine & 4 & 2 \\
\hline Olanzapine & 2 & 2 \\
\hline Oxcarbazepine & 4 & 2 \\
\hline Oxycodone & 2 & 2 \\
\hline Paroxetine & 5 & 2 \\
\hline Penfluridol & 0 & 2 \\
\hline Phenytoine & 5 & 2 \\
\hline
\end{tabular}

\begin{tabular}{|c|c|c|}
\hline Pimozide & 0 & 2 \\
\hline Pipamperone & 0 & 2 \\
\hline Posaconazole & 6 & 2 \\
\hline Promethazine & 4 & 2 \\
\hline Pseudoephedrine & 0 & $1 / 2 / 3$ \\
\hline Quetiapine & 4 & 2 \\
\hline Repaglinide & 5 & 2 \\
\hline Rilmenidine & 0 & 2 \\
\hline Risperidone & 4 & 2 \\
\hline Scopolamine & 2 & 2 \\
\hline Sertraline & 5 & 2 \\
\hline Sulpiride & 0 & 2 \\
\hline Tetrabenazine & 0 & 2 \\
\hline Tianeptine & 4 & 2 \\
\hline Tiapride & 0 & 2 \\
\hline Tramadol & 3 & 2 \\
\hline Trihexyphenidyle & 5 & 2 \\
\hline Trimipramine & 5 & 2 \\
\hline Tropatepine & 5 & 2 \\
\hline Venlafaxine & 4 & 2 \\
\hline Zuclopenthixol & 5 & 2 \\
\hline
\end{tabular}


TABLEAU VII : LiSTE DES MOLECULES D'INTERETS RECHERCHEES PAR SCREENING AVEC LEURS TEMPS DE RETENTION (TR) ET UN NIVEAU DE VIGILANCE 3.

\begin{tabular}{|c|c|c|}
\hline Analyte & $\begin{array}{l}\text { TR } \\
\text { théo }\end{array}$ & $\begin{array}{l}\text { Vigilance } \\
\text { conduite }\end{array}$ \\
\hline 6MAM & 2,3 & 3 \\
\hline 7-Aminoclonazepam & 3,2 & 3 \\
\hline Alphahydroxymidazolam & 4,3 & 3 \\
\hline Alprazolam & 4,9 & 3 \\
\hline Amphetamine & 2,2 & 3 \\
\hline Atropine & 2,7 & 3 \\
\hline Benzoylecgonine & 3,3 & 3 \\
\hline Bromazepam & 4,5 & 3 \\
\hline Clobazam & 4,9 & 3 \\
\hline Clonazepam & 4,7 & 3 \\
\hline Cocaethylene & 3,7 & 3 \\
\hline Cocaine & 3,4 & 3 \\
\hline Diazepam & 5,2 & 3 \\
\hline Doxylamine & 2,6 & 3 \\
\hline Ecgoninemethylester & 2 & 3 \\
\hline Estazolam & 5 & 3 \\
\hline Fluphenazine & 5 & 3 \\
\hline Haloperidol & 4 & $2 / 3$ \\
\hline Heroine & 3 & 3 \\
\hline Ketamine & 3 & 3 \\
\hline Lorazepam & 5 & 3 \\
\hline Lormetazepam & 5 & 3 \\
\hline LSD & 4 & 3 \\
\hline
\end{tabular}

\begin{tabular}{|c|c|c|}
\hline Analyte & $\begin{array}{l}\text { TR } \\
\text { théo }\end{array}$ & Vigilance conduite \\
\hline MBDB & 3 & 3 \\
\hline MDA & 2 & 3 \\
\hline MDEA & 3 & 3 \\
\hline MDMA & 2 & 3 \\
\hline Mephedrone & 3 & 3 \\
\hline Metamphetamine & 2 & 3 \\
\hline Midazolam & 4 & 3 \\
\hline Nitrazepam & 5 & 3 \\
\hline Nordiazepam & 5 & 3 \\
\hline Norketamine & 3 & 3 \\
\hline Oxazepam & 5 & 3 \\
\hline Prazepam & 6 & 3 \\
\hline Pseudoephedrine & 0 & $1 / 2 / 3$ \\
\hline Remifentanil & 0 & 3 \\
\hline Ropivacaine & 3 & 3 \\
\hline Sufentanyl & 4 & 3 \\
\hline $\mathrm{THC}$ & 6 & 3 \\
\hline $\mathrm{THC}-\mathrm{COOH}$ & 6 & 3 \\
\hline THC-COOH-glu & 6 & 3 \\
\hline $\mathrm{THC}-\mathrm{OH}$ & 6 & 3 \\
\hline Zolpidem & 4 & 3 \\
\hline Zopiclone & 3 & 3 \\
\hline
\end{tabular}

Dans le Tableau V, le terme «Vigilance conduite non défini » signifie que la molécule a été retenue pour ce travail comme pouvant présenter un impact potentiel sur la conduite, mais 
pour laquelle aucun des 3 niveaux de vigilance n'a été attribué (absence de pictogramme). Un niveau de vigilance 3 a été attribué systématiquement aux stupéfiants. Pour les métabolites de médicaments, le niveau de vigilance attribué est le même que celui de la molécule mère. Par exemple, pour le midazolam qui a un niveau 3, il a été attribué par défaut à son métabolite actif l'alphahydroxymidazolam un niveau 3, de même pour la venlafaxine et son métabolite actif la norvenlafaxine qui ont toutes les deux un niveau 2. Il en est de même pour les métabolites des stupéfiants qui, même lorsqu'ils sont inactifs et détectés isolément témoignent d'un usage récent du stupéfiant en question (ex. : la benzoylecgonine qui est un métabolite non psychoactif de la cocaïne mais qui présente une toxicité cardiovasculaire).

Les résultats ont été analysés au moyen du logiciel « Analyst ${ }^{\circledR}$ » permettant le retraitement des données et l'intégration des pics chromatographiques. Une représentation typique de ces données de retraitement est indiquée en Figure 18 (exemple d'un échantillon positif pour le citalopram au cours de cette étude).

Le premier chromatogramme (à gauche) présenté en Figure 18 correspond au blanc (solution dépourvue de l'analyte recherché), le second représente le signal obtenu pour l'échantillon de sang analysé, dilué au dixième, le troisième concerne l'échantillon pur, le quatrième correspond au calibrateur à une concentration de $10 \mathrm{ng} / \mathrm{mL}$ et le dernier correspond au calibrateur à $100 \mathrm{ng} / \mathrm{mL}$. On constate sur cette figure qu'il y a un pic dans le sang analysé, pur et dilué (graphiques 2 et 3), au temps de rétention et aux fragmentations caractéristiques du citalopram : $\operatorname{Tr}=4,1 \mathrm{~min}$ et $\mathrm{m} / \mathrm{z}: 325$ (ion père) $\rightarrow 109$ (ion fils ou fragment). La concentration estimée était de 124 ng/mL (Figure 17 : ligne 2 du tableau présenté). 


\begin{tabular}{|c|c|c|c|c|c|c|c|c|c|c|c|c|c|}
\hline & Sample Name & Sample ID & $\begin{array}{c}\text { Sample } \\
\text { Type }\end{array}$ & $\begin{array}{c}\text { Dilu } \\
\text { tion } \\
\text { Fac } \\
\text { tor }\end{array}$ & $\begin{array}{l}\text { Analyte Peak } \\
\text { Name }\end{array}$ & $\begin{array}{l}\text { Analyte } \\
\text { Peak Area } \\
\text { (counts) }\end{array}$ & $\begin{array}{l}\text { Analyte } \\
\text { Concen } \\
\text { tration } \\
(\mathrm{ng} / \mathrm{mL})\end{array}$ & $\begin{array}{l}\text { Analyte } \\
\text { Retention } \\
\text { Time } \\
\text { (min) }\end{array}$ & $\begin{array}{c}\text { Analyte } \\
\text { Mass } \\
\text { Ranges } \\
\text { (Da) }\end{array}$ & $\begin{array}{l}\text { Use } \\
\text { Rec } \\
\text { ord }\end{array}$ & $\begin{array}{l}\text { Rec } \\
\text { ord } \\
\text { Mod } \\
\text { ified }\end{array}$ & $\begin{array}{c}\text { Calculated } \\
\text { Concentration } \\
(\mathrm{ng} / \mathrm{mL})\end{array}$ & $\begin{array}{c}\text { Accuracy } \\
(\%)\end{array}$ \\
\hline 1 & BLBL-ST & Blanc ST-1602 & Blank & 1 & Citalopram & 0 & 0.00 & 0.0 & $325.200 /$ & & $\square$ & $\mathrm{N} / \mathrm{A}$ & $N / A$ \\
\hline 2 & 087.16-dilx10 & Thèse Maxime & Unknown & 10 & Citalopram & 5446000 & $\mathrm{~N} / \mathrm{A}$ & 3.9 & $325.200 /$ & & $\square$ & 124.00 & $\mathrm{~N} / \mathrm{A}$ \\
\hline 3 & 087.16 & Thèse Maxime & Unknown & 1 & Citalopram & 36007617 & $N / A$ & 3.9 & $325.200 /$ & & $\bar{\square}$ & 132.18 & $\mathrm{~N} / \mathrm{A}$ \\
\hline 4 & ST-10ng/ml & Dopants $10 \mathrm{ng} / \mathrm{ml}$ sur ST-1602 & Standard & 1 & Citalopram & 4833715 & 10.00 & 3.9 & $325.200 /$ & $\nabla$ & $\square$ & 10.00 & 100.0 \\
\hline 5 & ST-100ng/ml & Dopants $100 \mathrm{ng} / \mathrm{ml}$ sur ST-1602 & Standard & 1 & Citalopram & 27796468 & 100.00 & 3.9 & $325.200 /$ & $\bar{\nabla}$ & $\bar{\square}$ & 100.00 & 100.0 \\
\hline
\end{tabular}

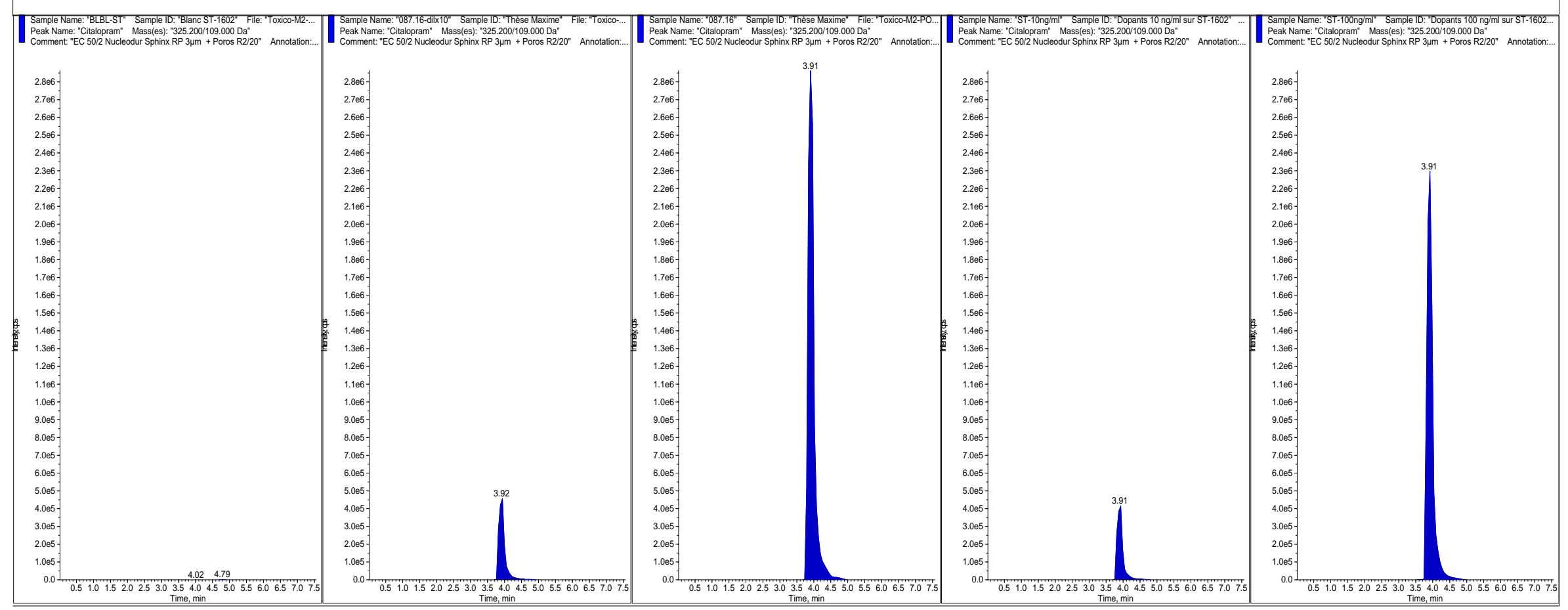

Figure 17 : TRACE CARACTERISTIQUE D'Un ECHANTILLON ANALYSE CONTENANT DU CITALOPRAM (NiVEAU DE VigiLANCE POUR A CONDUITE : 2). 


\subsection{Résultats}

\subsubsection{Caractéristiques démographiques des échantillons étudiés}

Les échantillons analysés se composaient de 100 individus. Le Tableau VIII présente les différentes classes d'âges de la population étudiée.

TABLEAU VIII : REPARTITION DES INDIVIDUS DE LA POPULATION ETUDIEE PAR CLASSE D'AGE.

\begin{tabular}{cc}
\hline Classes d'âges & Nombre d'individus \\
\hline$<20$ & 9 \\
$20-29$ & 37 \\
$30-39$ & 28 \\
$40-49$ & 11 \\
$50-59$ & 6 \\
$60-69$ & 7 \\
$70-79$ & 2 \\
\hline Total général & 100
\end{tabular}

Parmi la population étudiée on constate que toutes les classes d'âges sont représentées, avec des extrêmes allant de 18 jusqu'à 76 ans pour la personne la plus âgée. L’âge moyen étant de 33,7 ans +/- 14,2. Néanmoins, les 20-29 ans représentaient plus d'un tiers de l'échantillon (37\%), et les 30-39 ans en représentaient $28 \%$. La classe des $40-49$ ans comptait $11 \%$ des individus testés. La classe la moins représentée était celle des 70-79 ans avec seulement 2\%.

Le Tableau IX présente les pourcentages d'hommes et de femmes de la population étudiée.

TABLEAU IX : REPARTITION DES INDIVIDUS DE LA POPULATION ETUDIEE PAR SEXE.

\begin{tabular}{cc}
\hline Sexe & Nombre d'individus \\
\hline Homme & 90 \\
Femme & 10 \\
\hline Total & 100
\end{tabular}


Sur les 100 individus sélectionnées parmi ceux de l'année 2016, on constate une plus forte présence masculines avec $90 \%$ dans l'échantillon contre seulement $10 \%$ de femmes.

En ce qui concerne la répartition géographique des individus, celle-ci a été évaluée à partir de l'origine des réquisitions judicaires. Chaque brigade possédant un secteur géographique, il n'était pas possible de déterminer avec précision le lieu des faits, mais une estimation de la zone géographique concernée dans la région de Rouen a été réalisée (Figure 18).

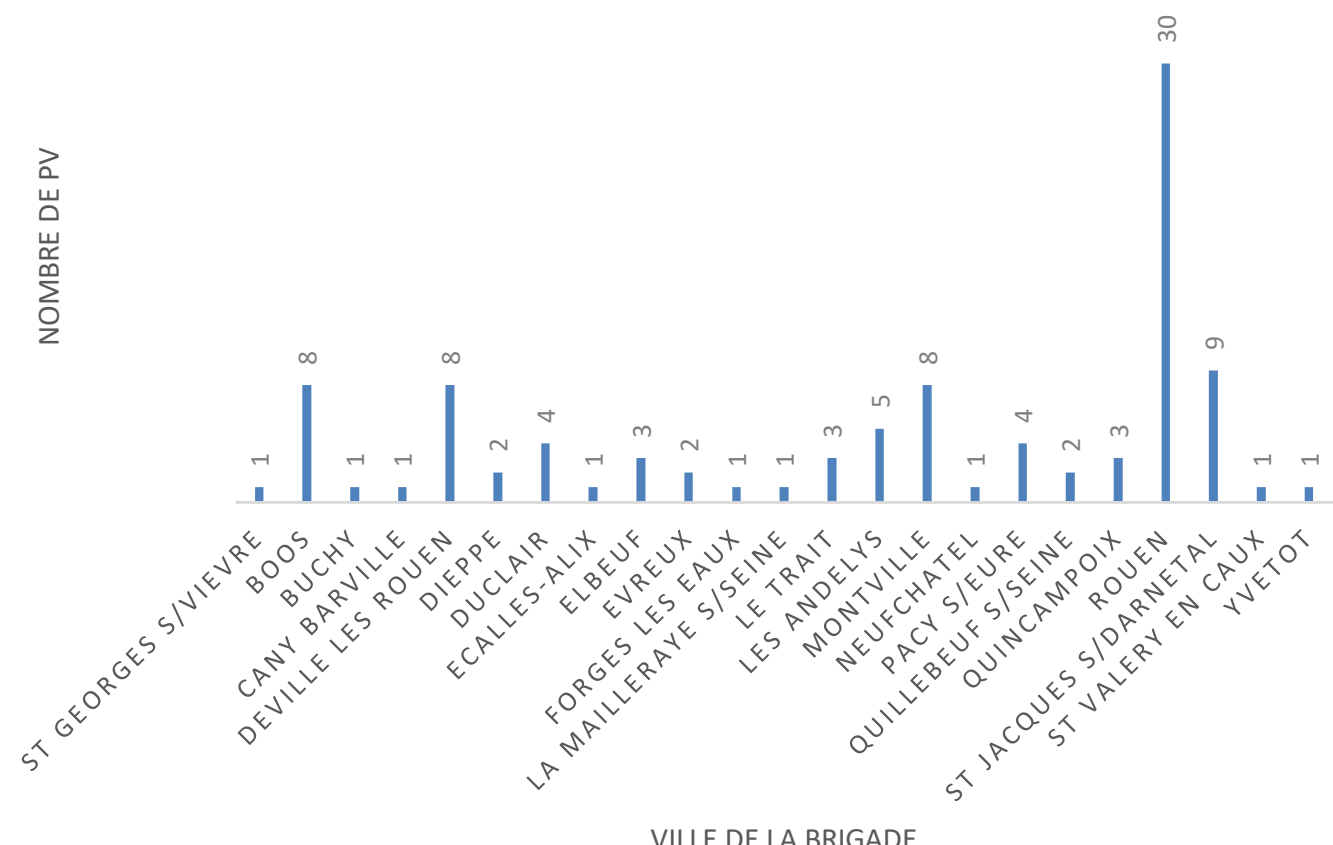

Figure 18 : Repartition des PRoces Verbaux (PV) EN FONCTION DE LEUR ORIGine GeOGRAPHiQue.

On constate dans la Figure 18 que la majorité des échantillons analysés proviennent d'accidents et de délits routiers autour de l'agglomération Rouennaise et de communes proches de Rouen (>60\%), dont Rouen (30\% des cas), St Jacques s/ Darnétal (9\%), Boos (8\%), Déville- 
lès-Rouen (8\%) ou Montville (8\%).Le reste des échantillons concerne d'autres brigades de gendarmerie, réparties dans la seine maritime et l'Eure (1 à 5\% des PV pour ces brigades).

En revanche l'ouest, l'extrême nord et sud de l'anciennement Haute-Normandie ne sont pas concernés par cette étude, puisque les prélèvements sont probablement effectués et analysés par d'autres laboratoires tels que celui de l'hôpital Jacques Monod du Havre, du CHU de Caen, d'Amiens ou encore de laboratoires situés en région parisienne.

2.4.2 Estimation de l'incidence des médicaments chez les conducteurs

Avant d'évaluer l'incidence, nous détaillons les résultats obtenus, la répartition des individus de la population étudiée en fonction de la consommation d'alcool et de stupéfiants associée ou non à une prise de médicaments.

L'alcool éthylique est recherché par CPG-FID. La Figure 19 représente l'incidence des cas positifs à l'éthanol dans la population de conducteurs étudiés (51\%), ainsi que la proportion de cas au cours desquels la recherche d'éthanol n'était pas requise ( $>31 \%)$. 


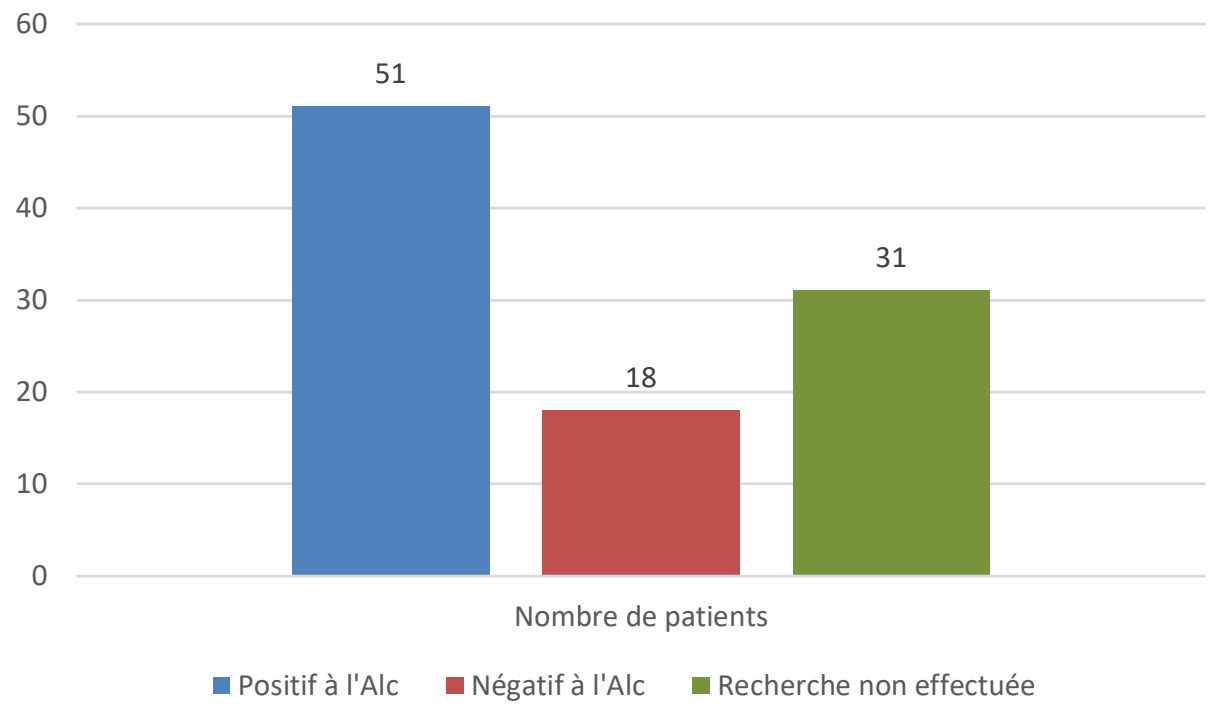

FIGURE 19 : REPARTITION DES INDIVIDUS DE L'ECHANTILLON EN FONCTION DE LA PRESENCE OU NON D'ETHANOL.

Pour les personnes positives, le seuil de positivité était de $0,1 \mathrm{~g} / \mathrm{L}$, limite de quantification de la méthode analytique utilisée. Le taux le plus faible quantifié était de 0,24 $\mathrm{g} / \mathrm{L}$ et le maximum était de $4,46 \mathrm{~g} / \mathrm{L}$.

La répartition en fonction des sexes était la suivante : sur 51 échantillons positifs, 47 étaient des hommes et 4 des femmes. Sur les 18 cas négatifs, on comptait 14 hommes et 4 femmes. Parmi les individus dont l'alcoolémie était non renseignée, on dénombrait 2 femmes contre 29 hommes.

Les résultats qualitatifs obtenus pour le THC sont présentés en figure 20. 


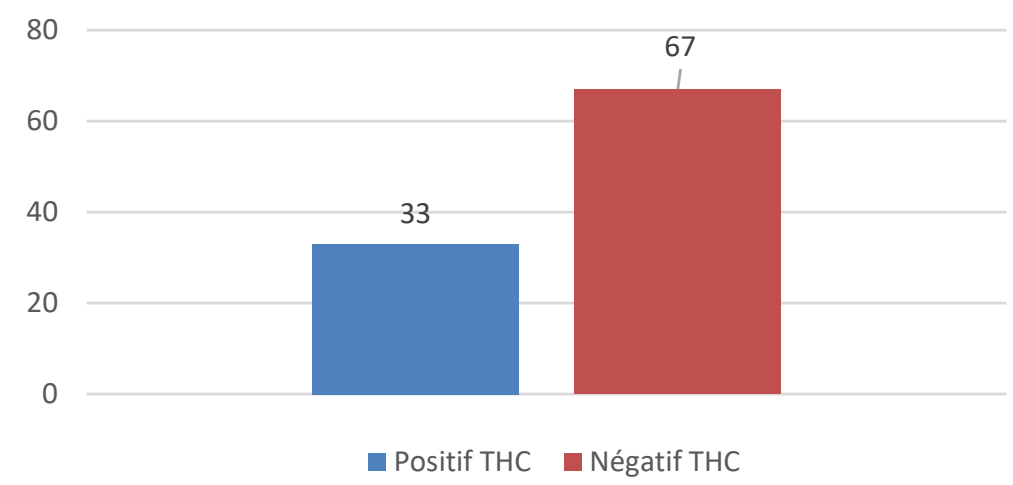

FIGURE 20 : REPARTITION DES INDIVIDUS DE L'ECHANTILLON EN FONCTION DE LA PRESENCE OU NON DE THC.

Un tiers des individus soit 33 ici étaient positifs au THC contre 67 négatifs.

TABLEAU X : REPARTITION DES CAS POSITIFS ET NEGATIFS EN THC EN FONCTION DU SEXE.

\begin{tabular}{cccc}
\cline { 2 - 4 } & Homme & Femme & Total \\
\hline Positif THC & 30 & 3 & 33 \\
Négatif THC & 60 & 7 & 67 \\
\hline Total & 90 & 10 & 100
\end{tabular}

D'après le tableau X, on constate que $30 \%$ de l'échantillon sont des hommes positifs au THC soit $1 / 3$ des hommes et donc $60 \%$ sont négatifs. 3\% de l'échantillon sont des femmes positives au THC et donc $7 \%$ sont des femmes négatives.

Les résultats des autres stupéfiants (amphétamines, cocaïne, héroïne, métabolites et dérivés) sont présentés en Figure 21. 


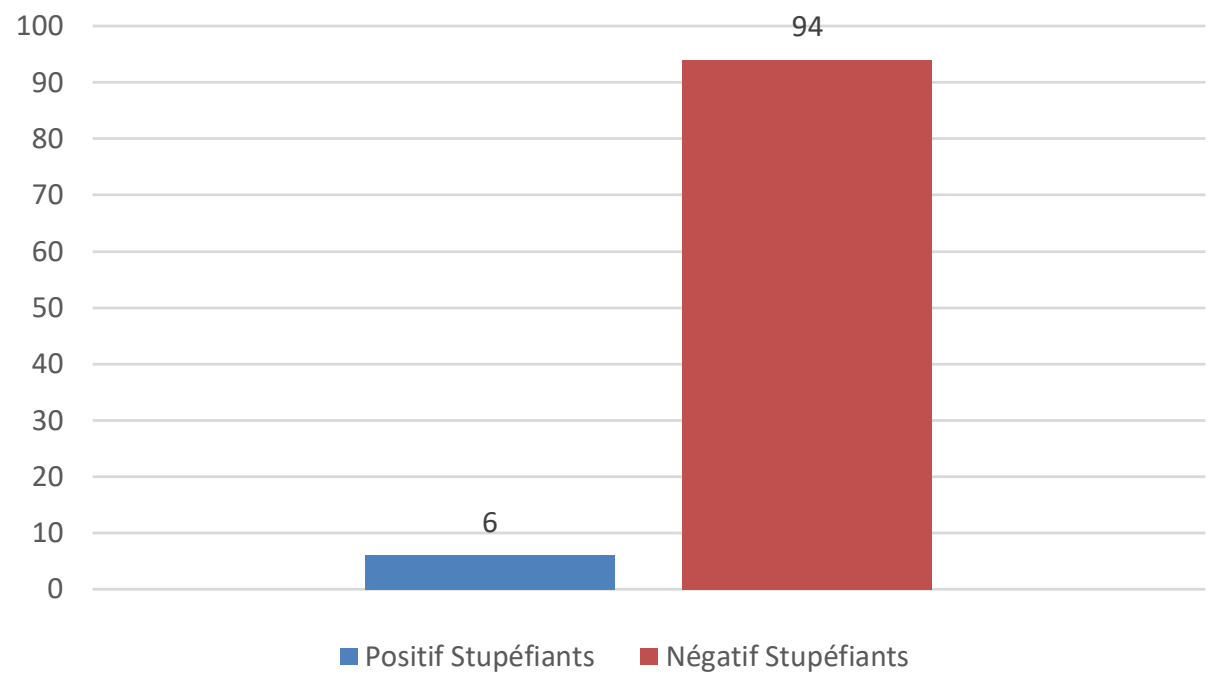

FIGURE 21 : REPARTITION DES INDIVIDUS ETUDIES EN FONCTION DE LA PRESENCE OU NON DE STUPEFIANTS.

La majorité des échantillons était négative pour ceux-ci. Soit 94 personnes négatives contre 6 personnes positives, dont la plupart étaient positives à la cocaïne.

TABLEAU XI : REPARTITION POSITIF/NEGATIF STUPEFIANTS EN FONCTION DU SEXE.

\begin{tabular}{cccc}
\cline { 2 - 4 } & Homme & Femme & Total \\
\hline Positif Stup & 5 & 1 & 6 \\
Négatif Stup & 85 & 9 & 94 \\
\hline Total & 90 & 10 & 100 \\
\hline
\end{tabular}

D’après le tableau XI, 85\% des individus étudiés étaient négatifs et représentés par des hommes, $9 \%$ sont des femmes négatives contre $1 \%$ de femmes positives et $5 \%$ sont des hommes positifs.

Les données concernant les médicaments sont présentés en figure 22. 
50

40

30

20

10

0
34

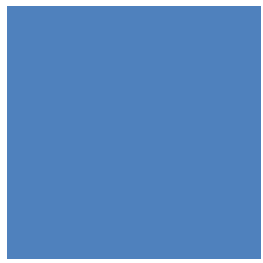

Positifs Mdts

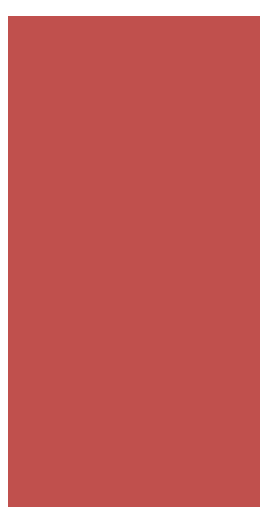

- Négatifs Mdts

FIgURE 22 : REPARTITION DES INDIVIDUS ETUDIES EN FONCTION DE LA PRESENCE OU NON DE MEDICAMENTS.

D’après les résultats ci-dessus, 34 personnes étaient positives aux médicaments (et/ou métabolites, prises individuelles ou prise en charge hospitalière) contre 66 personnes négatives, ce qui représente environ $1 / 3$ en données brutes.

TABLEAU XII : REPARTITION DES CAS POSITIFS ET NEGATIFS EN MEDICAMENT(S) EN FONCTION DU SEXE.

\begin{tabular}{cccc}
\hline & Homme & Femme & Total \\
\hline $\begin{array}{c}\text { Positif Médicament(s) } \\
\text { Négatif }\end{array}$ & 30 & 4 & 34 \\
Médicament(s) & 60 & 6 & 66 \\
\hline Total & 90 & 10 & 100 \\
\hline
\end{tabular}

D’après le Tableau XII, 30\% des conducteurs étudiés étaient des hommes positifs aux médicaments (soit $1 / 3$ des hommes) et $4 \%$ des femmes.

Les molécules retrouvées sont des médicaments mais aussi parfois leurs métabolites qui peuvent être actifs comme par exemple le diazépam qui possède comme métabolites le 
nordiazépam mais aussi l'oxazépam $\left(\right.$ Seresta $\left.^{\circledR}\right)$, et le témazépam, qui sont des métabolites actifs, commercialisés ou non.

TABLEAU II : FREQUENCE ET MOLECULES DE NIVEAU NON DEFINI RETROUVEES CHEZ LES CONDUCTEURS ETUDIES.

\begin{tabular}{ccc}
\hline Analyte & $\begin{array}{c}\text { Vigilance } \\
\text { conduite }\end{array}$ & $\begin{array}{c}\text { Nombre de } \\
\text { personnes }\end{array}$ \\
\hline Bisoprolol & Non défini & 2 \\
Indapamide & Non défini & 1 \\
\hline
\end{tabular}

TABLEAU III : FREQUENCE ET MOLECULES DE NIVEAU DE VIGILANCE 1 RETROUVEES CHEZ LES CONDUCTEURS ETUDIES.

\begin{tabular}{ccc}
\hline Analyte & $\begin{array}{c}\text { Vigilance } \\
\text { conduite }\end{array}$ & $\begin{array}{c}\text { Nombre de } \\
\text { personnes }\end{array}$ \\
\hline Amlodipine & 1 & 1 \\
Cetirizine & 1 & 2 \\
Codeine & 1 & 2 \\
\hline
\end{tabular}

TABlEAU XV : FREquenCE ET MOLECULES DE NIVEAU DE VIGILANCE 2 RETROUVEeS CHEZ LES CONDUCTEURS ETUDIES.

\begin{tabular}{ccc}
\hline Analyte & $\begin{array}{c}\text { Vigilance } \\
\text { conduite }\end{array}$ & $\begin{array}{c}\text { Nombre de } \\
\text { personnes }\end{array}$ \\
\hline Amitriptyline & 2 & 1 \\
Carbamazepine & 2 & 1 \\
Citalopram & 2 & 2 \\
EDDP & 2 & 1 \\
Fluoxetine & 2 & 1 \\
Gliclazide & 2 & 2 \\
Glimepiride & 2 & 1 \\
Hydroxyzine & 2 & 1 \\
Lamotrigine & 2 & 1 \\
\hline
\end{tabular}

\begin{tabular}{ccc}
\hline Analyte & $\begin{array}{c}\text { Vigilance } \\
\text { conduite }\end{array}$ & $\begin{array}{c}\text { Nombre de } \\
\text { personnes }\end{array}$ \\
\hline Levetiracetam & 2 & 1 \\
Lidocaine & 2 & 7 \\
Methadone & 2 & 1 \\
Morphine & 2 & 10 \\
Norfluoxetine & 2 & 1 \\
Norvenlafaxine & 2 & 1 \\
Paroxetine & 2 & 2 \\
Promethazine & 2 & 1 \\
Venlafaxine & 2 & 2 \\
\hline
\end{tabular}




\begin{tabular}{ccc}
\hline Analyte & $\begin{array}{c}\text { Vigilance } \\
\text { conduite }\end{array}$ & $\begin{array}{c}\text { Nombre de } \\
\text { personnes }\end{array}$ \\
\hline Alphahydroxymidazolam & 3 & 4 \\
Benzoylecgonine & 3 & 4 \\
Bromazepam & 3 & 1 \\
Diazepam & 3 & 1 \\
Ketamine & 3 & 4 \\
\hline
\end{tabular}

\begin{tabular}{ccc}
\hline Analyte & $\begin{array}{c}\text { Vigilance } \\
\text { conduite }\end{array}$ & $\begin{array}{c}\text { Nombre de } \\
\text { personnes }\end{array}$ \\
\hline Nordiazepam & 3 & 1 \\
Norketamine & 3 & 3 \\
Oxazepam & 3 & 3 \\
Zolpidem & 3 & 1 \\
Midazolam & 3 & 4 \\
\hline
\end{tabular}

Certaines molécules présentes dans les tableaux XV et XVI sont métabolites comme la cocaïne avec la benzoylecgonine qui peut-être aussi utilisé comme principe actif dans une solution topique pour les douleurs musculaires $\left(\right.$ Esterom $\left.^{\circledR}\right)$, mais aussi des médicaments classés comme stupéfiant (c'est-à-dire avec une législation particulière de prescription, dispensation) ou l'EDDP un métabolite de la méthadone. Chez certains patients, seul le métabolite d'un médicament était détecté (comme la venlafaxine et son métabolite la norvenlafaxine), et témoignait d'une administration relativement ancienne par rapport aux faits, même si celui-ci est actif. Reprenons l'exemple du diazépam qui est fortement métabolisé par le foie majoritairement en desméthyldiazépam actif (demi-vie de 30 à 150 heures). Ce métabolite est lui-même transformé par hydroxylation en oxazépam et en témazépam,également actifs, qui sont par la suite inactivés par glucuroconjugaison (33).

Un score a été établi afin d'identifier les individus étant sous l'influence de plusieurs substances psychoactives. Dans ce score, une personne positive à l'alcool correspond à 3 points et si négative 0 point, de même pour les stupéfiants 3 points par classe de stupéfiant si positive et 0 si négative. Pour les médicaments le nombre de points attribué correspond au niveau du pictogramme : un niveau $1=1$ point, un niveau $2=2$ points et un niveau $3=3$ points. Pour les médicaments à niveau non défini il a été attribué 0,5 points. Lorsque plusieurs médicaments étaient détectés pour un même individu, les points étaient additionnés. 


\begin{tabular}{cc}
\hline Score & Nombre de personnes \\
\hline $0 \leq X<0,5$ & 8 \\
$0,5 \leq X<1$ & 1 \\
$1 \leq X<1,5$ & 0 \\
$1,5 \leq X<2$ & 0 \\
$2 \leq X<2,5$ & 5 \\
$2,5 \leq X \leq 3$ & 55 \\
$>3$ & 31 \\
\hline Total & 100
\end{tabular}

Dans le tableau XVII, on constate que 8 personnes présentaient un score nul, c'est-àdire négatif à toutes les substances, une personne avait un score de 0,5 (positive à un médicament de niveau de vigilance non défini), et la majorité de la population étudiée a un score $\leq 3$ soit 55 individus. Cette catégorie représente les personnes ayant consommé plusieurs médicaments (plutôt de niveau de vigilance faible à 2 maximum). Néanmoins, 31 personnes possèdent un score $>3$, ce qui signifie une probable association entre l'alcool et/ou stupéfiants et/ou médicaments, ou encore une variété plus importante de médicaments, on retrouve: 6 associations médicaments + stupéfiants (environ 19,5\%), 3 stupéfiants + alcools (9,5\%), 6 présentaient une association uniquement médicamenteuse (19,5\%), 4 associaient médicaments + alcools + stupéfiants $(13 \%)$ et enfin 12 associations de médicaments + alcool (38,5\%). MEDICAMENTS UNIQUEMENT) OBTENUS CHEZ LES CONDUCTEURS ETUDIES.

\begin{tabular}{cc}
\hline Score & Nombre de personnes \\
\hline $0 \leq X<0,5$ & 66 \\
$0,5 \leq X<1$ & 2 \\
$1 \leq X<1,5$ & 1 \\
$1,5 \leq X<2$ & 0 \\
$2 \leq X<2,5$ & 10 \\
$2,5 \leq X<3$ & 9 \\
$>3$ & 12 \\
\hline Total & 100 \\
\hline
\end{tabular}


D’après le Tableau XVIII, on constate que la grande majorité de la population étudiée était négative pour les médicaments (66 / 100), deux individus étaient positifs pour des médicaments de niveau non défini $(0,5 \leq \mathrm{X}<1)$, un seul positif à un médicament de niveau 1 $(1 \leq \mathrm{X}<1,5)$, pour le reste soit l'individu est positif à un médicament de niveau 2, soit il a consommé plusieurs médicaments de niveaux différents soit $31 \%$ des cas.

En ne gardant que les molécules correspondantes à des spécialités médicamenteuses lors de cette étude, 26 molécules sont mises en évidence. Néanmoins certains de ces médicaments correspondaient vraisemblablement à des médicaments administrés par les services d'urgence au cours de la prise en charge thérapeutique suite à un accident, comme par exemple la kétamine ou le midazolam. Après avoir consulté le dossier médical des personnes sélectionnées, il a été possible de mettre en évidence une prise en charge hospitalière pour certaines avec administration médicamenteuse, permettant ainsi de les exclure de l'analyse puisque les molécules retrouvées ne sont pas la cause de l'accident ou du délit routier (administration $a$ posteriori lors de la prise en charge médicale).

Pour les 34 individus positifs aux médicaments, le dossier médical a été vérifié pour ceux présentant des traces de midazolam, kétamine, morphine et métabolites associés afin de vérifier qu'ils aient bien reçu ces produits lors de leur prise en charge médicale. Au final seulement 22 molécules correspondantes à des spécialités pharmaceutiques ont été retenues dans l'analyse (+/- éthanol et produits stupéfiants) (figure 23). 


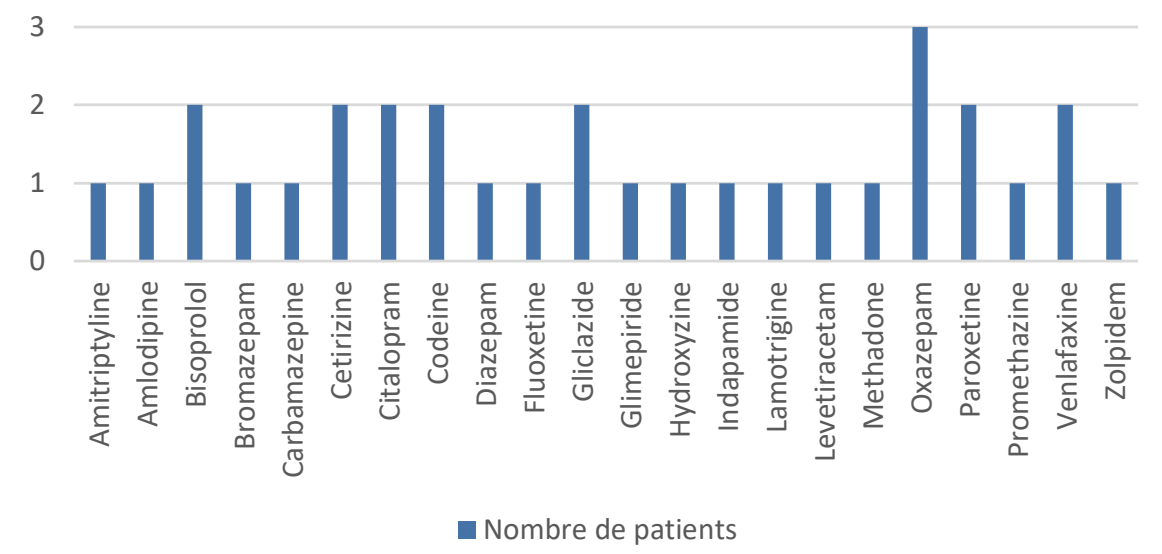

FIGURE 23 : HISTOGRAMME REPRESENTANT LES MOLECULES ET LEUR FREQUENCE CHEZ LES CONDUCTEURS ETUDIES.

La plupart des individus étaient positifs à plusieurs médicaments (par exemple un même conducteur était positif au gliclazide, à la paroxétine et au zolpidem) et sur un échantillon de 100 personnes, 17 personnes soit près de $20 \%$ avaient consommé des substances médicamenteuses susceptibles d'avoir un impact sur leur conduite automobile, pouvant entrainer un accident de la route et/ou un délit routier.

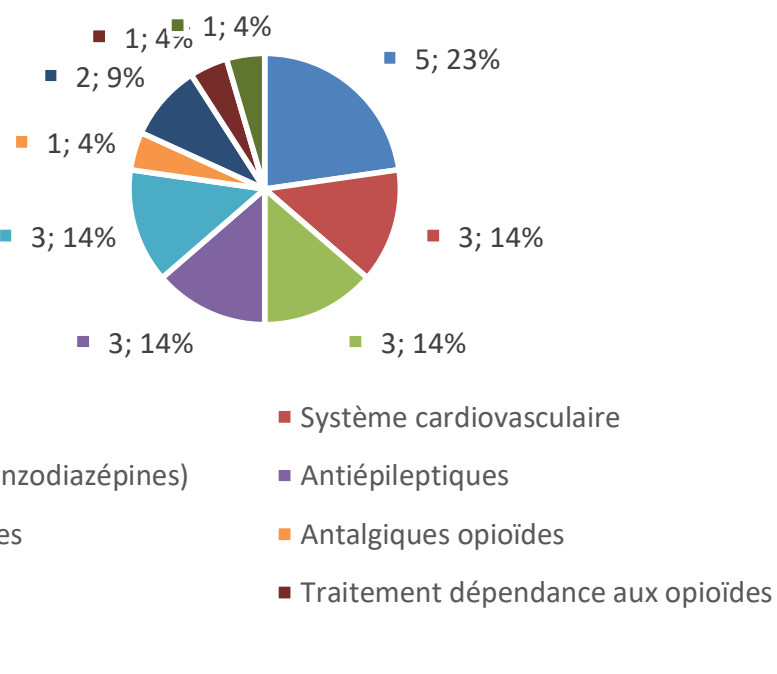

FIGURE 24 : GRAPHIQUE REPRESENTANT LA REPARTITION DES MEDICAMENTS CHEZ LES INDIVIDUS ETUDIES. 
D'après la Figure 24, parmi les 17 individus et sur les 22 molécules retenues, 23\% (soit 5 molécules) sont des antidépresseurs, suivis par les anxiolytiques (benzodiazépines), les antihistaminiques, les antiépileptiques et des médicaments du système cardiovasculaire à 14\% chacun (soit 3 molécules) puis les antidiabétiques 9\% (soit 2 molécules) et enfin les hypnotiques, les antalgiques opioïdes et les traitements de la dépendance aux opioïdes à $4 \%$ chacun (soit 1 molécule).

En regroupant les médicaments du système nerveux, il ressort un total de 59\% (soit 13 molécules). L'hydroxyzine est un cas particulier, appartenant aux antihistaminiques. Ce médicament est couramment utilisé comme anxiolytique / sédatif si on le rajoute aux médicaments du système nerveux, ce groupe passe à 14 molécules soit environ $63 \%$ des médicaments.

Plus de la moitié des médicaments (63\%) ayant eu un potentiel impact sur la capacité à conduire sont des médicaments du système nerveux (hydroxyzine compris). Sur les 17 individus finaux positifs, 11 avaient consommé cette catégorie de médicaments, ce qui représente environ $65 \%$ des cas positifs.

Au final, l'incidence des médicaments chez les conducteurs d'engins motorisés dans un contexte d'accidents de la route et de délits routiers sur notre série de cas au CHU de Rouen en 2016 est de $17 \%$. 


\section{Discussion}

L'objectif de ce travail était d'évaluer l'impact potentiel des médicaments sur la conduite d'engins motorisés, et il convient de prendre en compte certains biais liés à cette étude.

D’une part, l'effectif de l'échantillon étudié était relativement limité, ici 100 individus avec une répartition homme / femme déséquilibrée même si la sélection a été effectuée au hasard dans un « pool » de conducteurs.

De plus la population de cette échantillon a été ciblée dès l'inclusion puisqu'il s'agit d'individus ayant fait l'objet d'une demande d'analyses par les forces de l'ordre, suite à un accident de la route et/ou un délit routier, associé ou non à un dépistage rapide. Ainsi, il existe dans cette population «à risque » une probabilité accrue de sélectionner des personnes positives pour différentes substances psychoactives (alcool, drogues, médicaments). En d'autres termes l'incidence était potentiellement plus importante que dans la population générale.

D'autre part, certains sujets étaient positifs du fait de médicaments administrés lors de leur prise en charge médicale (avec administration de morphine, midazolam le plus souvent) via les services d'urgences ou le SAMU. Ces individus ont donc été écartés de l'analyse.

De plus, certaines molécules présentant potentiellement des effets sur la conduite d'engins n'ont pas été recherchées dans cette étude, par exemple les médicaments antiparkinsoniens (ceux-ci ne faisant pas l'objet de suivi thérapeutique en pratique courante, ils sont rarement recherchés dans le cadre de screenings toxicologiques). Les substances recherchées avec la méthode utilisée au cours de cette étude correspondent à celles le plus couramment retrouvées dans la population dans des contextes d'intoxications volontaires ou non, ou dans des contextes de surdosages en suivi thérapeutique. 
Par ailleurs, l'aspect galénique n'a pas été pris en compte au cours de ce travail. En effet, certains médicaments peuvent avoir un impact plus important sur la conduite selon leur mode d'administration (collyre, intraveineux, etc.). La majorité des collyres possèdent des pictogrammes de vigilance d'au moins niveau 2, puisque leur administration est directement faite sur l'œil et entraine une gêne, plus ou moins temporaire, de la vue et a ainsi un impact direct sur la capacité à conduire des engins motorisés. Par exemple la spécialité Mydriaticum ${ }^{\circledR}$ (tropicamide) utilisée pour les examens ophtalmologiques contient une molécule atropinique entrainant une mydriase. Ceci expose à une plus grande susceptibilité à l'éblouissement, rendant difficile la conduite automobile et l'utilisation d'engins motorisés.

Nous pouvons aussi nous interroger sur l'attribution de certains niveaux de pictogrammes, en effet certains antalgiques comme la codéine, la morphine, ne sont que des niveaux 2 or on sait que ces molécules peuvent avoir un fort potentiel sédatif, ainsi que dépresseur respiratoire sur certaines personnes ou en association. Ne faudrait-il pas, par simple principe de précautions dès lors qu'il y une forte variable interindividuelle appliquer le pictogramme de niveau supérieur, pour plus de vigilance?

Malgré ces biais que nous avons tenté de prendre en compte, les résultats de ce travail nous permettent de mettre en évidence une incidence des médicaments « à risque » dans près de $20 \%$ des automobilistes testés. Cette fréquence est élevée et n'est généralement pas considérée lors de contrôles automobiles, alors que certaines classes thérapeutiques pourraient présenter une synergie délétère avec des comportements à risque tels que les consommations d'alcool et/ou de produits stupéfiants. Ces résultats laissent préjuger d'une incidence considérable des médicaments dans la population générale. L’information des patients apparait donc nécessaire et le rôle de conseil du pharmacien d'officine pourrait limiter les risques liés à la consommation de certains médicaments sur la conduite automobile. 


\section{Conclusion}

Les résultats obtenus au cours de ce travail montrent une réelle incidence des médicaments dans les accidents de la route et les délits routiers. Cela est d'autant plus vrai si ces molécules sont couplées avec la prise d'autres substances psychoactives telles que l'alcool et les drogues licites et illicites.

Au-delà la prévention et de la répression des facteurs de risque comme la vitesse, l'alcool et les drogues, il serait probablement utile d'étendre la prévention routière aux médicaments. Un travail d'éducation auprès de la population sur l'utilisation des médicaments et leurs risques sur la conduite automobile et l'utilisation d'engins motorisés semble nécessaire. L'impact des pictogrammes apposés sur le conditionnement secondaire des médicaments s'est progressivement banalisé au cours des années suivant leur instauration. Une resensibilisation de la population pourrait permettre d'enrayer ce phénomène. Le conseil pharmaceutique apparait comme une étape primaire indispensable à la sensibilisation de la population directement concernée, mais il est également possible de renforcer l'information au cours de journées de préventions routières organisées par les forces de l'ordre auprès de publics variés (écoliers, étudiants, motards, etc.), ou des campagnes d'informations via le Cespharm ou l'Ordre des Pharmaciens.

La coordination entre médecins et pharmaciens pourrait également jouer un véritable rôle d'éducation thérapeutique, au cabinet comme en pharmacie d'officine, pour sensibiliser les patients lors de la prescription et de la dispensation des médicaments. Les différents effets indésirables des médicaments, leur impact sur la vigilance, la prise en compte du terrain pathologique du patient mais aussi des associations médicamenteuses prescrites permettraient d'adapter au mieux les schémas posologiques en fonction du mode de vie du patient. Il pourrait 
en résulter une réduction des risques sur la conduite automobile parleur prise en compte dès la mise en place du traitement. 


\section{Références bibliographiques}

1. Observatoire national Interministériel de la sécurité routière. Accidentalité routière 2017 - estimations au 25 janvier 2018 [Internet]. Observatoire national Interministériel de la sécurité routière; 2018 [cité 8 févr 2018]. Disponible sur: http://www.securiteroutiere.gouv.fr/la-securite-routiere/l-observatoire-national-interministeriel-de-lasecurite-routiere

2. Observatoire national Interministériel de la sécurité routière. Drogues [Internet]. Sécurité routière | Tous responsables. [cité 8 févr 2018]. Disponible sur: http://www.securiteroutiere.gouv.fr/dangers-de-la-route/drogues

3. Larousse É. Définitions : médico-légal, médico-légale, médico-légaux - Dictionnaire de français Larousse [Internet]. [cité 8 févr 2018]. Disponible sur: http://www.larousse.fr/dictionnaires/francais/m\%C3\%A9dico1\%C3\%A9gal_m\%C3\%A9dico-1\%C3\%A9gale_m\%C3\%A9dico-1\%C3\%A9gaux/50130

4. Cavalié P, Djeraba A. Analyse des ventes de médicaments en France en 2013. ANSM; 2014 juin p. 36.

5. Lafortune G, Biondi N, Paris V, Belloni A, Morgan D, Mueller M, et al. Panorama de la santé 2015 | OECD READ edition [Internet]. OECD iLibrary. [cité 15 févr 2018]. Disponible sur: http://www.keepeek.com/Digital-Asset-Management/oecd/social-issuesmigration-health/panorama-de-la-sante-2015_health_glance-2015-fr

6. Ministère de la Santé. Code de la santé publique - Article R5121-139 [Internet]. Code de la santé publique. Disponible sur: https://www.legifrance.gouv.fr/affichCodeArticle.do?cidTexte=LEGITEXT0000060726 65\&idArticle=LEGIARTI000006914891 \&dateTexte $=\&$ categorieLien $=$ cid

7. Afssaps. Informations sur les médicaments et la conduite automobile. 2005.

8. Ministère de la Santé, Delaporte S. Arrêté du 8 août 2008 pris pour l'application de l'article R. 5121-139 du code de la santé publique et relatif à l'apposition d'un pictogramme sur le conditionnement extérieur de certains médicaments et produits.

9. Orriols L, Luxcey A, Contrand B, Gadegbeku B, Delorme B, Tricotel A, et al. Road traffic crash risk associated with benzodiazepine and z-hypnotic use after implementation of a colour-graded pictogram: a responsibility study. $\mathrm{Br} \mathrm{J}$ Clin Pharmacol. déc 2016;82(6):1625-35.

10. Centre Régional de Pharmacovigilance et d'Informations sur le médicament de RouenNormandie. Viking. 2017.

11. Sécurité Routière, Ordre des Pharmaciens, ANSM. LA SÉCURITÉ SUR LA ROUTE COMMENCE SUR VOTRE TABLE DE NUIT. AFFICHE.

12. Sécurité Routière, Ordre des Pharmaciens, ANSM. LA SECURITE SUR LA ROUTE COMMENCE SUR VOTRE TABLE DE NUIT. BROCHURE. 
13. Ministère de l'intérieur, Barbe E, Ministère de la Santé, Vallet B. Arrêté du 16 décembre 2017 modifiant l'arrêté du 21 décembre 2005 modifié fixant la liste des affections médicales incompatibles avec l'obtention ou le maintien du permis de conduire ou pouvant donner lieu à la délivrance de permis de conduire de durée de validité limitée.

14. Larousse É. Définitions : psychoactif - Dictionnaire de français Larousse [Internet]. [cité 15 févr 2018]. Disponible sur: http://www.larousse.fr/dictionnaires/francais/psychoactif/10910008

15. Alcool info service. Culture de l'alcool en France : Consommation de vin et promotion alcoolinfoservice [Internet]. Alcool Info Service. [cité 13 mai 2018]. Disponible sur: http://www.alcool-info-service.fr/alcool/consommation-alcool-france/culture-alcoolconsommation-vin

16. Association Nationale de Prévention en Alcoologie et Addictologie. Alcool [Internet]. [cité 13 mai 2018]. Disponible sur: http://www.anpaa.asso.fr/sinformer/addictionslessentiel-sur/alcool

17. Alcool Info Service. Le binge-drinking ou « biture express » c'est quoi ? alcoolinfoservice [Internet]. Alcool Info Service. [cité 13 mai 2018]. Disponible sur: http://jeunes.alcool-info-service.fr/alcool/binge-drinking

18. Observatoire national Interministériel de la sécurité routière. Les chiffres de l'alcool [Internet]. Sécurité routière | Tous responsables. [cité 8 févr 2018]. Disponible sur: http://www.securite-routiere.gouv.fr/medias/les-chiffres-de-la-route/les-chiffres-de-lalcool

19. Larousse É. Définitions : stupéfiant - Dictionnaire de français Larousse [Internet]. [cité 24 mai 2018]. Disponible https://www.larousse.fr/dictionnaires/francais/stup\%C3\%A9fiant/74948

20. Larousse É. Définitions : drogue - Dictionnaire de français Larousse [Internet]. [cité 24 mai 2018]. Disponible sur: https://www.larousse.fr/dictionnaires/francais/drogue/26827

21. Drogues info service. Qu'est-ce qu'une drogue? [Internet]. Drogues Info Service. [cité 24 mai 2018]. Disponible sur: http://www.drogues-info-service.fr/Tout-savoir-sur-lesdrogues/Les-drogues-et-leur-consommation/Qu-est-ce-qu-une-drogue

22. Beck F, Richard J-B, Guignard R, Le Nézet O, Spilka S. Les niveaux d'usage des drogues en France en 2014 [Internet]. OFDT; 2015 mars [cité 14 janv 2018] p. 8. Report No.: 99. Disponible sur: https://www.ofdt.fr/BDD/publications/docs/eftxfbv3.pdf

23. Cadet-Taïrou A, Milhet M. Les usages détournés de médicaments codéinés par les jeunes [Internet]. OFDT; 2017 [cité 14 janv 2018]. Disponible sur: https://www.ofdt.fr/BDD/publications/docs/eisxacx7v2.pdf

24. Ministère de la Santé, Buzyn A. Arrêté du 12 juillet 2017 portant modification des exonérations à la réglementation des substances vénéneuses. juill 12, 2017.

25. Milhet M, Langlois E. Jeunes et médicaments psychotropes [Internet]. OFDT; 2016 mai [cité 14 janv 2018] p. 4. Disponible sur: https://www.ofdt.fr/BDD/publications/docs/eftxmmw5.pdf 
26. ANSM. Médicaments à risque d'usage détourné ou de dépendance - ANSM : Agence nationale de sécurité du médicament et des produits de santé [Internet]. ANSM. [cité 7 juin 2018]. Disponible sur: http://ansm.sante.fr/Activites/Surveillance-des-stupefiants-etdes-psychotropes/Medicaments-a-risque-d-usage-detourne-ou-de-dependance/(offset)/0

27. Observatoire national Interministériel de la sécurité routière. Accidentalité routière 2017 - résultats définitifs. Observatoire national Interministériel de la sécurité routière; p. 9.

28. Sécurité Routière. Drogues [Internet]. Sécurité routière| Tous responsables. [cité 7 juin 2018]. Disponible sur: http://www.securite-routiere.gouv.fr/connaitre-lesregles/reglementation-et-sanctions/drogues

29. Mura P, Kintz P, Ludes B, Gaulier J., Marquet P, Martin-Dupont S, et al. Comparison of the prevalence of alcohol, cannabis and other drugs between 900 injured drivers and 900 control subjects: results of a French collaborative study. Forensic Sci Int. 2003;79-85.

30. Ministère de la justice, Ministère de l'action et des comptes publiques. Arrêté du 29 septembre 2017 pris en application du $4^{\circ}$ de l'article 2 du décret $n^{\circ} 2017-248$ du 27 février 2017 relatif aux modalités de fixation du tarif des actes prescrits dans le domaine de la médecine légale, de la psychologie légale, de la toxicologie, de la biologie et de la radiologie et relevant des frais de justice | Legifrance [Internet]. Disponible sur: https://www.legifrance.gouv.fr/eli/arrete/2017/9/29/JUSB1725836A/jo/texte/fr

31. Ministère de la justice, Chaleh-Marzban P. Note $n^{\circ}$ SJ-17-374-FIP4/06.11.2017 [Internet]. 2017 [cité 15 août 2018]. Disponible sur: http://www.cnbae.org/CirculaireDSJ2017toxicologie.pdf

32. Biotechnologie \& Biologie et Physiopathologie humaine, Académie de Rouen. Biotechnologie \& Biologie et Physiopathologie humaine - Académie de Rouen - HPLC Principe et appareillage [Internet]. [cité 17 août 2018]. Disponible sur: http://biotech.spip.ac-rouen.fr/spip.php?article9

33. ANSM, Laboratoire Arrow. Résumé des Caractéristiques du Produits DIazépam comprimé Arrow [Internet]. [cité 25 sept 2018]. Disponible sur: http://agenceprd.ansm.sante.fr/php/ecodex/frames.php?specid=61437096\&typedoc=R\&ref=R032285 1.htm 


\section{ULENIVERSITÉ}

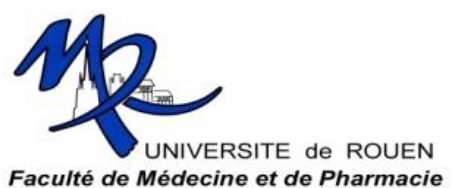

SERMENT DE GALIEN

Je jure d'honorer ceux qui m'ont instruit dans les préceptes de mon art et de leur témoigner ma reconnaissance en restant fidèle à leur enseignement.

D'exercer dans l'intérêt de la Santé publique ma profession avec conscience et de respecter non seulement la législation en vigueur mais aussi les règles de l'Honneur, de la Probité et du Désintéressement.

De ne jamais oublier ma responsabilité et mes devoirs envers le malade et sa dignité humaine.

De ne dévoiler à personne les secrets qui m'auraient été confiés ou dont j'aurais eu connaissance dans l'exercice de ma Profession.

En aucun cas, je ne consentirai à utiliser mes connaissances et mon état pour corrompre les mœurs et favoriser les actes criminels.

Que les hommes m'accordent leur estime si je suis fidèle à mes promesses.

Que je sois méprisé de mes Confrères si je manque à mes engagements.

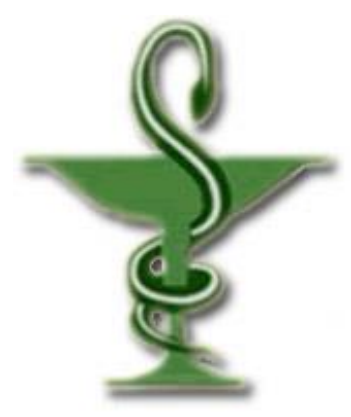




\section{Maxime THIOLLLENT}

Quelle incidence des médicaments sur les accidents de la route et sur les délits routiers ? Analyse d'une série de cas au CHU de Rouen.

Th. D. Pharm., Rouen, 2018, 62p.

\section{RESUME}

La sécurité routière est un enjeu majeur de la société actuelle. L'objectif de la lutte contre l'insécurité routière est notamment de faire diminuer le nombre d'accidents et de morts sur les routes, en augmentation en 2017 avec 61570 accidents et environ 3700 décès. Parmi les facteurs de risque connus, les consommations d'alcool et de produits stupéfiants présentent un impact important. L'impact de la consommation de médicaments susceptibles de réduire les capacités à la conduite automobile reste sous-évalué.

Au cours de ce travail nous avons évalué l'incidence des consommations d'alcool éthylique, de produits stupéfiants et/ou de substances médicamenteuses « à risque », dans une série de 100 automobilistes ayant fait l'objet de prélèvements sanguins au décours d'un contrôle routier ou d'accidents de la circulation. Ces échantillons biologiques ont fait l'objet d'un screening toxicologique par HPLC-MS/MS.

Parmi les 90 hommes et 10 femmes inclus dans cette étude, 51\% présentaient une alcoolémie positive [0,24-4,46 g/L]. Dans 33\% des cas un dépistage des cannabinoïdes (THC) était positif, les autres stupéfiants étaient retrouvés dans $6 \%$ des échantillons analysés (cocaïne principalement) et dans $17 \%$ des cas le dépistage d'un (ou plusieurs) médicament(s) susceptible(s) d'impacter la conduite automobile était positif. Ces médicaments incluaient principalement des psychotropes (anxiolytiques, hypnotiques, antidépresseurs), des traitements de substitution aux opiacés, des antiépileptiques et des médicaments à visée cardiovasculaire. Malgré un biais de sélection évident de la population de conducteurs étudiée, l'incidence importante des médicaments retrouvés laisse préjuger d'une incidence importante dans la population générale. Le recours à des pictogrammes informatifs apparait aujourd'hui banalisé et le renforcement du conseil pharmaceutique en officine pourrait probablement contribuer à réduire les risques associés à la prise de certains médicaments chez les automobilistes.

MOTS CLES : Conduite automobile, médicaments, alcool, stupéfiants, toxicologie

\section{JURY}

Président du jury : Professeur Michel GUERBET, PU

Membres du jury : Docteur Fabien LAMOUREUX, PH

Docteur Marc PONTHIEUX, Pharmacien d'officine

Docteur Laurent IMBERT, PH

DATE DE SOUTENANCE : 19 décembre 2018 